\title{
PFC/JA-85-19
}

\section{Line-tying of Interchange Modes \\ in a Hot Electron Plasma}

\author{
M.J. Gerver and B.G. Lane \\ October 1985 \\ Plasma Fusion Center \\ Massachusetts Institute of Technology \\ Cambridge, MA 02139
}

Submitted for publication in: Bhysics of Fluids 
Abstract

We have solved the dispersion relation for low frequency $\left(\omega \ll \omega_{c i}\right)$ electrostatic flutelike interchange modes in a mirror cell with a fraction $\alpha$ of hot electrons. With bulk line-tying to cold (non-emitting) end walls, using a sab model and the local approximation. It is found that hot electron interchange modes are more effectively stabilized by line-tying than MHD interchange modes, because 1) the line-tying is enhanced by a factor $\left(\omega / \nu_{e}\right)^{1 / 2}$ when the wave frequency $\omega$ is greater than the cold electron collision frequency $\nu_{\theta}$; and 2) hot electron interchange modes can be completely stabilized, rather than merely having their growth rates reduced. if there is a spread of hot electron curvature drift velocities. Predictions of the minimum $\alpha$ needed for instability and of the first azimuthal mode number in to go unstable, and of the scaling of these quantities with neutral gas pressure, are in good quantitative agreement with observations of hot electron interchange instabilities in the Tara tandem mirror experiment, provided a correction is made for the fact that the modes in Tara are not flutelike, but should have higher amplitude in the plug than in the central cell. The theory may also explain observations in $E B T$ and TMX-U. Increasing the ion temperature $T_{i}$ should have a modest stabilizing effect. In addition to the hot electron interchange modes, there are also ion driven interchange modes, which are unstable even in the absence of hot electrons, but which generally have low growth rates, much 
less than MHD growth rates. Even these modes may be completely stabilized by FLR and line-tying when $T_{i}$ is sufficiently great. 


\section{Introduction}

Stability to interchange ${ }^{1}$ and trapped particle modes ${ }^{2}$ is an important requirement for the success of tandem mirrors. In order to avoid resonant radial transport, ${ }^{3}$ it is desirable for a tandem mirror to be completely axisymmetric, or at least to have the non-axisymmetric anchor cells outside the plug cells ("outboard anchors") 80 that most particles in the central cell see only axisymmetric magnetic fields. 4 But axisymmetric tandem mirrors necessarily have average bad curvature and should be unstable to interchange modes, while outboard anchors allow the possibility of unstable trapped particle modes localized in the axisymetric central cell and plugs.

The problem is exacerbated in tandem mirrors with thermal barriers, 5 which require hot electrons to be localized in plug cells of high mirror ratio (and hence of especially bad curvature, if they are axisymmetric). Unstable interchange modes (or trapped particle modes) driven by these hot electrons might be expected to occur in the plugs of the Tara tandem mirror, which has outboard anchors, and indeed such modes have been observed in Tara, ${ }^{6}$ as well as in EBT?

Hot electron interchange modes differ from the usual interchange modes described by magnetohydrodynamics, in that their frequency $\omega$ can be less than or comparable to the hot electron curvature drift frequency $\omega_{\text {deh }}$. When this happens, the hot electrons become decoupled from the mode, which 
becomes a stable negative energy precessional mode. The linear theory of these modes was first worked out (in the electrostatic limit and for $\omega \ll$ $\omega_{c 1}$ ) by Krall, "who found that they become stable when the fraction $\alpha$ of hot electron $\left(\alpha \equiv n_{\text {hot }} / n_{e}\right)$ is below a critical value $\alpha_{c \text { rit }}=m^{2} \tau_{e h} / 4 m_{i} R_{p} R_{c} \omega_{c i}{ }^{2}$, where In is the azimuthal mode number. $\mathrm{T}_{\text {eh }}$ is the hot electron temperature. $R_{p}$ is the plasma radius, $R_{c}$ is the radius of curvature, $\omega_{c i}$ is the ion cyclotron frequency, and $m_{i}$ is the ion mass. Note that this critical $\alpha$ is an increasing function of $m$; as the fraction of hot electrons rises starting from a low initial value, we would expect that the modes of lowest In would go unstable first.

In a typical run of Tara, the plug is heated by ECRH, and a component of hot electrons $\left(T_{e h} \simeq 200 \mathrm{keV}\right.$ ) is formed in the plug, as well as a component of cold electrons $\left(T_{e c} \simeq 40 \mathrm{eV}\right)$ in the plug and in the central cell. The cold electron density is sufficient to stabilize hot electron interchange modes, and as long as the ECRH is on, no hot electron interchange modes are observed. When the ECRH is turned off, the hot electrons density in the plug falls very slowly (with a decay time of about $10^{-1} \mathrm{sec}$ ) because the hot electrons have a very low collisional loss rate and cooling rate, but the cold electron density falls more rapidly (with a decay time on the order of $10^{-3} \mathrm{sec}$ ). When the cold electron density has dropped to some fraction of its original value, an instability is observed 
in the plug and central cell which has phase velocity comparable to the hot electron curvature drift velocity, and which causes rapid radial losses of hot electrons. This instability is presumed to be the hot electron interchange mode, but in one respect its behavior differs from what we would expect on the basis of the simple theory outlined above; the first mode to go unstable is not at low m, but at m $=9$ to 15 .

We have found that this behavior can be explained by line-tying. which can stabilize the low m modes. The earliest theoretical studies of linetying ${ }^{9}$ ignored the possible existence of a potential sheath at the end walls and found that complete stabilization of interchange modes would occur if there was a sufficient density of cold plasma between the mirror throats and the end walls. This result was used to explain the stability of several early mirror experiments which had average bad curvature. ${ }^{10}$ Perkins and Post 11 pointed out that this theory would only be applicable if the end walls were hot enough to emit a secondary electron current as great as the end loss current of electrons from the plasma; if the end walls were cold (as they would be in devices like Tara, in which the field lines fan out by a large factor before they reach the end walls), they would develop a sheath which would inhibit line-tying. In this case, Kunkel and Guillory ${ }^{12}$ showed that line-tying could not stabilize interchange modes completely, but only reduce their growth rates, from $\gamma_{\text {mhd }}$ to $\frac{\gamma_{\text {mhd }}^{2} k^{2} T_{\text {ec }}}{m_{i} \omega_{c i}^{2} \nu_{\text {loss }}}$, where $\nu_{\text {loss }}$ is the 
axial loss rate. Although this kind of line-tying was invoked to explain the relative stability of some early mirror experiments ${ }^{13}$. in other experiments line-tying has been ineffective unless the end walls are hot enough to eliminate the sheath. ${ }^{14}$ For Tara, this kind of line-tying would only cause a modest reduction in growth rate for the lowest m modes, and would not explain why the low II modes are not seen at all. Furthermore, line-tying through a sheath gives rise to a positive dissipative term in the dispersion relation, and would actually de-stabilize the negative energy precessional mode ${ }^{15}$ (at high m) for arbitrarily small hot electron fraction. The preceding analysis applies to line-tying of MHD interchange modes. There are two reasons why line-tying can be more effective in stabilizaing hot electron interchange modes, and can thus explain the behavior of Tara. The first reason (which can also be applicable to MED interchange modes) is that the mode frequency $\omega$ is often greater than the electron collision frequency $\nu_{e}$. a situation which Kunkel and Guillory ${ }^{12}$ did not consider. When this happens, the line-tying term is enhanced by a factor $\left(i \omega / \nu_{e}\right)^{1 / 2}$ (compared to the case $\omega \ll \nu_{e}$ for the same $\nu_{\text {loss }}$ ), because only a thin layer of electrons near the loss boundary in velocity space is able to respond to the perturbed potential. For hot electron interchange modes in Tara, $\omega \simeq$ $10^{6} \sec ^{-1}$ and $\nu_{e} \simeq 10^{4} \sec ^{-1}$, so the line-tying term is enhanced by a factor of 10. This results in a substantial reduction in growth rate, but still would not completely stabilize the hot electron interchange mode. 
The second reason is that the hot electrons will not all have the same curvature drift velocity, but will have a spread of drift velocities, due to their spread in $v_{\perp}$ and $v_{\|}$. The hot electron term in the dispersion relation will then have an imaginary part (coming from the resonant denominator $\omega-\omega_{d}$ ) which acts like a negative dissipative term in the dispersion relation. If the line-tying is strong enough to bring the growth rate for the hot electron interchange mode down to the drift frequency for a typical hot electron. then this negative dissipation will start to become comparable to the positive dissipation of the line-tying term, and (in contrast to the situation where all hot electrons have the same drift velocity), the negative energy wave can become completely stable, rather than just having its growth rate reduced.

When these two effects $\left(\omega>\nu_{e}\right.$ and the spread in hot electron drift velocities) are taken into account, we find that even line-tying to cold end walls can stabilize the hot electron interchange modes at lower m in Tara, leaving intermediate values of m (comparable to the observed values $m \simeq 9$ to 15) as the first modes to go unstable as the hot electron fraction $\alpha$ increases. In addition to explaining the Tara results, it has been suggested that line-tying may play a stabilizing role in $T M X-U^{16}$. It has been found that when radially segmented floating end plates are used in TMX$U$. radial transport is somewhat reduced, because the radial electric field is reduced. 17 However, the effect is not very dramatic: the radial 
confinement time is only increased by a factor of 1.5. It is possible that. when the end plates are grounded. line-tying stabilizes interchange or trapped particle modes, but that these go unstable (causing increased radial transport) when the end plates are floated (and line-tying cannot occur between field lines going to different plates): this de-stabilizing effect of the floating end plates could almost cancel out the stabilizing effect of reducing the radial electric field. Line-tying was also used as an explanation for the stability of Elmo (the predecessor of EBT) ${ }^{18}$, and it may explain the fact ${ }^{7}$ that the first hot electron interchange mode to go unstable in EBT (which has parameters somewhat similar to Tara) is at $m=7$. rather than at low m. (However, the instability seen in EBT has $\omega>\omega_{c i}$, so the results of this paper are not directly applicable to EBT; instead, it would be necessary to look at line-tying of the high frequency hot electron interchange mode of Berk. ${ }^{19}$ )

In order to understand the essential features of line-tying of hot electron interchange modes, we have used a model (see Fig. 1) consisting of an isolated axisymetric mirror cell (such as the Tara plug and central cell) with grounded conducting end walls at either end, outside the mirror throats. The mode is assumed to be flute-like through the length of the plasma, with the perturbed potential falling to zero (its value at the end walls) in the sheaths near the end walls. The radial and azimuthal variation in the perturbed potential is treated using the local 
approximation. $i . e$. WKB is used and $k$ is taken to be entirely in the azimuthal direction, and the radial density scale length is taken as a constant $R_{p}$. The modes are assumed to be electrostatic (there is no coupling between shear and compressional motion of the magnetic field lines $^{20}$, and the $\nabla B$ drift is assumed to be that of the vacuum magnetic field; these assumptions are justified if $\beta$ is low enough.

of course this model is a great oversimplification of what is happening in Tara. In particular, the modes in Tara are probably not flute-like. but have higher amplitude in the plug (where the drive is), although there is evidence that the mode amplitude is not zero in the central cell. Also, the main contribution to line-tying is probably from the central cell (which has a greater end loss current than the plug), and $\nu_{\text {loss }}$ in the central cell is a steep function of radius (being much greater near the plasma edge), so the local approximation may not be valid. Finally, (especially at low $\mathrm{I}$ ) the mode may extend into the anchor, which is not axisymetric, and which could stabilize interchange modes without line-tying. Despite the simplified nature of our model, the numerical results we have found (e.g. for the first II to go unstable as the the hot electron fraction $\alpha$ increases) are in good quantitative agreement with observations in Tara, if some corrections are made based on a reasonable guess for the axial variation of the mode amplitude. Although this agreement may be somewhat fortuitous, our results do demonstrate that line-tying should play an important role in determining the behavior of interchange instabilities in the Tara plug and in 
experiments with comparable parameters, such as TMX-U and EBT, and they Indicate the need for further calculations using more realistic models. Such calculations are currently in progress.

In Sec. II, the dispersion relation for interchange modes is derived. It describes two branches of modes that can be unstable: hot electron driven modes, and ion driven modes. It is shown that in order for a mode to be completely stabilized by line-tying, both positive and negative dissipation are needed; if the dissipative terms only have one sign, then all mode numbers will be unstable.

In Sec. III, the marginal stability condition (hot electron fraction $\alpha$ as a function of mode number $m$ ) is found for the hot electron driven modes. Expressions are given for $\alpha$ crit, the minimum $\alpha$ at which any $m$ is unstable, and for mcrit. the first II to go unstable as $\alpha$ reaches $\alpha$ crit. The effect of higher ion temperature on these modes is considered, and ion FLR is seen to have only a modest stabilizing effect, in the sense that higher ion temperature only causes a modest increase in $\alpha_{\text {crit }}$.

In Sec. IV, the ion-driven mode is examined. For Tara plug parameters, it is shown to be unstable, but with a very low growth rate, much less than $\gamma_{\text {mhd, } i} \equiv\left(T_{i} / m_{i} R_{p} R_{c}\right)^{1 / 2}$ As $T_{i}$ increases, the growth rate remains low and the mode may become completely stable (due to ion FLR) when $T_{i}$ is sufficiently 
great (about $20 \mathrm{keV}$ ). The ion driven mode may have been seen in Tara, ${ }^{6}$ but if so it saturates at a low level and does not have much effect on confinement.

Section $V$ presents a summary and conclusions, and discusses some features which could be added to the model to make it more realistic. 


\section{Dispersion Relation}

We use a square well slab model for the mirror cell, with a uniform magnetic field $B_{O}$ in the z-direction, rising abruptly to $B_{\max }$ at the mirror throats and with gradients in density and magnetic field (and curvature of field lines) in the $x$-direction. We consider electrostatic perturbations with $k_{x}=0$ (because we are using the local approximation) and $k_{z}=0$ :

$$
\phi^{(1)}(x, y, z, t)=\phi^{(1)} \exp (i k y-i \omega t)
$$

The plasma consists of ions, a cold electron component (which does the linetying but does not contribute to the drive) and a hot electron component (which does not contribute to line-tying). Because these modes are quasineutral, the dispersion relation is of the form

$$
\rho_{\text {ec }}^{(1)}+\rho_{\text {eh }}^{(1)}+\rho_{i}^{(1)}=0
$$

where $\rho_{e c}^{(1)}, \rho_{e h}^{(1)}$, and $\rho_{i}^{(1)}$ are respectively the perturbed charge densities of cold electrons, hot electrons and ions. Each species is assumed to have a bimaxwellian distribution 


$$
f_{s 0}\left(v_{\perp} \cdot v_{\|}\right)=\frac{\exp \left(-v_{\perp}^{2} / 2 v_{s \perp}^{2}-v_{||}^{\left.2 / 2 v_{s}^{2} \mid\right)}\right.}{(2 \pi)^{3 / 2} v_{s \perp}^{2} v_{s||}}
$$

If desired, a different distribution function could be expressed as a sum of several bimaxwellian components of different temperatures and densities (including negative densities to represent a loss cone, for example) ${ }^{21}$ so that the results derived here will be easily generalizable. But we do not expect the behavior of these modes to be very sensitive to the details of the distribution function.

The perturbed charge density for each species is

$$
\rho_{s}^{(1)}=\tilde{\rho}_{s}^{(1)}-\frac{i}{\omega} \nabla \cdot J_{\text {loss, s }}^{(1)^{*}}
$$

Here $\tilde{\rho}_{s}^{(1)}$ is the usual expression for perturbed charge density in a low frequency electrostatic flute wave in the local approximation 22

$$
4 \pi \tilde{\rho}_{s}{ }^{(1)}=\phi_{\phi}^{(1)} \frac{\omega_{\mathrm{ps}}^{2}}{v_{\mathrm{s} \perp}^{2}} \cdot\left[1-\int \mathrm{dv} \mathrm{f}_{s 0}\left(v_{\perp}, v_{||}\right) J_{0}^{2}\left(k v_{\perp} / \omega_{c s}\right) \frac{\omega-\omega_{* s}}{\omega-\omega_{\mathrm{ds}}\left(v_{\perp}, v_{||}\right)}\right]
$$


where $\omega_{p s}$ and $\omega_{c s}$ are the plasma frequency and cyclotron frequency for species $s, \omega_{* s}$ is the diamagnetic drift frequency

$$
\omega_{* s} \equiv-\operatorname{sgn}\left(q_{s}\right) k v_{s \perp}^{2} / R_{p} \omega_{c s}
$$

$\left(R_{p}\right.$ being the density gradient scale length $n|d n / d x|^{-1}$ and $q_{s}$ being the charge of species $s)$, and $\omega_{d s}\left(v_{\perp}, v_{\|}\right)$is the drift in the $y$ direction due to $\nabla B$ and curvature. Since we are assuming $\nabla B$ is given by the vacuum field, we have

$$
\omega_{\mathrm{ds}}\left(v_{\perp} \cdot v_{\|}\right)=k\left(\frac{v_{L}^{2}}{2}+v_{\|}^{2}\right) R_{c}^{-1} \omega_{c s}^{-1}
$$

where $R_{c}$ is the radius of curvature. The quantity $J_{l o s s, s}^{(1)}$ appearing in Eq. (4) is the perturbation in the loss current due to the wave. We.consider only end losses (not radial losses), so

$$
\nabla \cdot J_{\text {loss,s }}^{(1)}=\frac{d}{d z} J_{\text {Ioss, s }}^{(1)} \simeq \frac{1}{L} J_{\text {Ioss, s }}^{(1)}
$$

We have assumed that the hot electrons have no end losses. Although the equilibrium ion endloss current is equal in magnitude to the equilibrium 
cold electron endloss current, we neglect the ion contribution to the perturbed end loss current, because the wave frequency $\omega$ is usually much greater than the ion bounce frequency. Then the dispersion relation is

$$
4 \pi \tilde{\rho}_{e h}^{(1)}+4 \pi \tilde{\rho}_{i}^{(1)}+4 \pi \tilde{\rho}_{e c}^{(1)}-\frac{4 \pi i}{\omega L} J_{\text {Ioss }}^{(1)}=0
$$

where the first three terms are the usual dispersion relation for low frequency hot electron interchange modes, and the last term is the linetying term.

To evaluate the $\tilde{\rho}_{s}^{(1)}$ terms, we change the variables in the velocity space integral in Eq. (5) from $v_{\perp}$ and $v_{\|}$to $u \equiv v^{2} / 2 v_{s \perp}^{2}$ and $\omega_{\text {ds }}$ (using the definition in Eq. (7)). The $\omega_{\text {ds }}$ integration can be done analytically, in terms of the Fried-Conte $\mathrm{Z}$ function. ${ }^{23}$ In order to do the $\mathrm{u}$ integration, we define a function

$$
G(a, b, c) \equiv \int_{0}^{\infty} d u e^{-u} J_{0}^{2}(b \sqrt{u})(a-c u)^{-1 / 2} z(\sqrt{a-c u})
$$


where $\sqrt{a-c u}$ is taken to have a positive imaginary part (which corresponds to taking the correct Landau contour in doing the $\mathrm{v}_{\mid}$integral in Eq. (5)). Then,

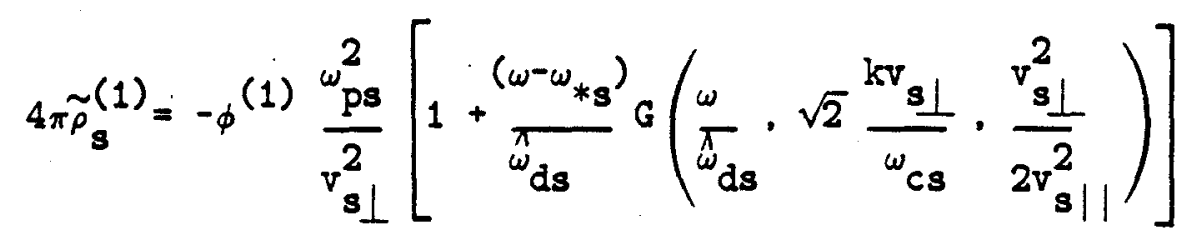

where $\hat{\omega}_{\mathrm{ds}} \equiv 2 k v_{s||^{2}}^{/ R_{c} \omega_{c s}}$.

For the cold electrons, we have $\omega / \hat{\omega}_{\mathrm{ds}} \rightarrow \infty$ and $\mathrm{kv}_{\mathrm{s} \perp} / \omega_{\mathrm{cs}} \rightarrow 0$. Then, using

$$
\underset{a}{\lim } \rightarrow \infty(a, 0, c)=-a^{-1}
$$

(which easily follows from Eq. (10)), Eq. (11) yields

$$
4 \pi \tilde{\rho}_{\mathrm{ec}}^{(1)}=-\frac{\omega_{\mathrm{pec}}^{2} k}{\omega_{c e^{\omega R} \mathrm{p}}} \phi{ }^{(1)}-\frac{k^{2} \omega_{\mathrm{pec}}^{2}}{\omega_{c e}^{2}} \phi(1)
$$

The second term in Eq. (13), the electron polarization drift (or inertia) term, is small, and will henceforth be neglected.

The hot electron term takes a particularly simple form if $\mathrm{T}_{1}=2 \mathrm{~T}_{1}$, so for convenience we will assume this. We can also let $\mathrm{kv}_{s \perp} / \omega_{c s} \rightarrow 0$ for the hot electrons. i.e. we can neglect hot electron FLR effects. (At least this 
is true for the hot electron temperatures typical of Tara; for EBT, hot electron FLR effects may start to become important, and perhaps should be included.) Then, using the identity

$$
G(a, 0,1)=-z^{\prime}(\sqrt{a})
$$

we find

$$
4 \pi \tilde{\rho}_{\mathrm{eh}}^{(1)}=-\frac{\omega_{\mathrm{peh}}^{2}}{\mathrm{R}_{\mathrm{p} \omega_{\mathrm{ce}}{ }^{\omega} \mathrm{deh}}} \mathrm{Z}^{\prime}\left(\sqrt{\omega / \hat{\omega}_{\mathrm{deh}}}\right)_{\phi}^{(1)}
$$

For the ions, at least for lower ion temperatures, we can take $\omega / \hat{\omega} \mathrm{ds}$ $\gg 1$ and $\mathrm{kv}_{\mathrm{s} \perp} / \omega_{\mathrm{cs}} \ll 1$. Taking an asymptotic expansion of $\mathrm{G}$ for large a and a power series for small b,

$$
G(a, b, c)=-a^{-1}+\frac{1}{2} b^{2} a^{-1}-\left(c+\frac{1}{2}\right) a^{-2}
$$

we find

$$
4 \pi \tilde{\rho}_{i}^{(1)}=\left[\frac{k \omega_{p i}^{2}}{R_{p} \omega \omega_{c i}}-\frac{k^{2} \omega_{p i}^{2}}{\omega_{c i}^{2}}-\frac{k^{2}\left|\omega_{* i}\right| \omega_{p i}^{2}}{\omega \omega_{c i}^{2}}-\frac{k^{2} \omega_{p i}^{2} \gamma_{\text {mhd,i }}^{2}}{\omega_{c i}^{2} \omega^{2}}\right] \phi(1)
$$


In deriving Eq. (17), we have adopted the convention that $\omega_{* i}<0$ and $\omega_{* e}$ $>0$, so that wares propagating in the electron diamagnetic direction will have $\omega / k>0$, and waves propagating in the ion diamagnetic direction will have $\omega / x<0$.

The terms on the right hand side of Eq. (17) are respectively the radial $E^{(1)} \times B_{O}$ convection term, the ion polarization drift (inertia) term, the ion FLR term, and the ion MHD drive term. If there were no hot electrons, no line-tying, and no ion FLR, then the cold electron radial convection term (the first term on the right hand side of Eq. (13), which is the only cold electron term we have kept) would exactly cancel the ion radial convection term, and the dispersion relation. Eq.. (9), would consist only of the ion polarization drift and ion drive terms, yielding

$$
\omega^{2}=-\gamma_{\text {mhd,i }}^{2} \equiv-\frac{\left(v_{i \perp}^{2}+v_{i||}^{2}\right)}{2 R_{p} R_{c}}
$$

Another limit which is sometimes appropriate for the ions is $\omega / \omega_{\mathrm{di}} \rightarrow \infty$ but $k v_{i \perp} / \omega_{c i}$ of order unity. Then, using

$$
\lim _{a \rightarrow \infty} G(a, b, c)=-a^{-1} \exp \left(-b^{2} / 2\right) I_{0}\left(b^{2} / 2\right)
$$


we find

$$
4 \pi \tilde{\rho}_{i}^{(1)}=\frac{-\omega_{\mathrm{p} i}^{2}}{v_{i \perp}^{2}}\left[1-\left(1+\frac{\left|\omega_{* i}\right|}{\omega}\right) \exp \left(\frac{-\mathrm{k}^{2} v_{i \perp}^{2}}{\omega_{c i}^{2}}\right) I_{0}\left(\frac{k^{2} v_{i \perp}^{2}}{\omega_{c i}}\right)\right]_{\phi}^{(1)}
$$

This expression is used when the ion Larmor radius is comparable to or greater than a wavelength, and $\omega \gg \hat{\omega}_{\mathrm{d} i}$.

To find the perturbed electron endloss current $J_{\text {loss }}^{(1)}$ which is needed for the line-tying term in the dispersion relation, we first calculate the steady state endloss current associated with the equilibrium potential. If the plasma were at the same potential as the end wall, then the equilibrium collisional endloss rate of cold electrons would usually exceed the loss rate of ions; hence the plasma develops a sufficiently large positive potential (generally a few times the cold electron temperature) to make the loss rates equal. (It is assumed that the end walls are cold, so they do not emit any electrons). To find the equilibrium endloss rate of electrons for a given potential, one must find the magnitude of the source term $S$ needed to make

$$
\frac{d f_{0}}{d t}=s(\underset{\sim}{v})+\underset{\sim}{F} \cdot \frac{\partial f_{0}}{\partial \underset{\sim}{v}}+\frac{\partial}{\partial \underset{\sim}{v}} \cdot \underset{\approx}{D} \cdot \frac{\partial f_{0}}{\partial \underset{\sim}{v}}=0
$$


(where $f_{0}(v)$ is the equilibrium electron distribution function, $\underset{\sim}{F}$ is the collisional drag and $\underset{\approx}{\mathbb{D}}$ is the collisional velocity diffusion tensor) subject to the boundary condition $f_{0}(v)=0$ at

$$
\epsilon-\mu \mathrm{B}_{\text {wall }}-e \Phi=0
$$

Here $\epsilon=\frac{1}{2} m_{e} v^{2}, \mu=\frac{1}{2} m_{e} v^{2} / B_{O}$, and $\Phi$ is the potential drop from the plasma to the wall. This boundary condition is appropriate if the electron bounce frequency is much greater than the collision frequency. Equation (21) can be solved analytically in certain limits by the method of Pastukhov, or it can be solved numerically. ${ }^{25}$ Typically one finds

$$
\int \mathrm{dv} S(\underset{\sim}{v}) \equiv \nu_{\text {loss }} \simeq \nu_{\theta} \exp \left(-e \Phi / T_{\text {ec }}\right)
$$

where $\nu_{e} \equiv \pi n_{e c} e^{4}(\ln \alpha) m_{e}^{-1 / 2} T_{e c}^{-3 / 2}$, lnd is the Coulomb logarithm. $T_{e c}$ is the cold electron temperature, and $\mathrm{n}_{\mathrm{ec}}$ is the cold electron density. This implies

$$
\mathrm{J}_{\text {loss }} \simeq \operatorname{Len}_{e c} \nu_{\theta} \exp \left(-\mathrm{e} \Phi / \mathrm{T}_{\text {ec }}\right)
$$


If the potential $\Phi$ is perturbed slightly by a wave at a frequency $\omega$ that is low compared to the collision rate $\nu_{e}$. then the distribution function $f_{e}$ will have time to adjust itself, at each phase of the wave, to be the solution to Eq. (21) satisfying the perturbed boundary condition, $f_{e}(v)=0$ at

$$
\epsilon-\mu \mathrm{B}_{\text {waIl }}-e[\Phi+\phi(1) \exp (i k y-i \omega t)]=0
$$

and the perturbed endloss current $J_{10 s 8}^{(1)}$ can be found by taking the derivative with respect to $\Phi$ of the equilibrium $\mathrm{J}_{\text {Ioss }}$ given by Eq. (24). Then

$$
\mathrm{J}_{\text {loss }}^{(1)}=\frac{\partial \mathrm{J}_{\text {loss }}}{\partial \Phi} \phi^{(1)} \simeq-\frac{\mathrm{Le}^{2} \mathrm{n}_{e c} \nu_{\text {loss }}}{\mathrm{T}_{e c}} \phi
$$

In. Eq. (26), we have neglected a term $e^{-1}\left(\partial J_{\text {loss }} / \partial n_{\text {ec }}\right) \tilde{\rho}_{\text {ec }}$, which is justified ${ }^{26}$ if $\omega \gg \nu_{\text {loss }}$. The line-tying term in the dispersion relation, Eq. (9), is then 12

$$
\frac{4 \pi i}{\omega L} J_{\text {loss }}^{(1)} \simeq \frac{-i \omega_{\operatorname{pec}}^{2} \text { loss }}{\omega v_{e c}^{2}} \phi^{(1)}
$$

A more rigorous calculation of $\mathrm{J}_{\text {loss }}^{(1)}$ would show ${ }^{26}$ that the right hand side of Eq. (27) should be multiplied by a factor $\left(1-\omega_{* e c} / \omega\right)$. 
If the perturbation in $\Phi$ occurs at a frequency $\omega>\nu_{e}$, then the distribution function does not have time to reach a new equilibrium satisfying Eq. (21) at each phase of the perturbation. Instead, the perturbation in $f_{e}$ is confined to a narrow region of velocity space near the loss boundary (given by Eq. (22)). The width of this boundary layer is the distance in velocity space over which the electrons can diffuse in one wave period, viz.

$$
\Delta v \simeq\left(T_{e c} / m_{e}\right)^{1 / 2}\left(\nu_{e} / \omega\right)^{1 / 2}
$$

Well within this boundary layer, the collisional loss rate is much greater than w. so Eq. (21) is satisfied subject to the boundary condition

$$
f_{e}(\underline{\sim}) \equiv f_{0}(\underline{v})+f_{e}^{(1)}(\underline{v})=0
$$

at the perturbed loss boundary, given by Eq. (25). Since the unperturbed distribution function $f_{0}(\underline{\sim})=0$ at the unperturbed loss boundary, given by Eq. (22), and since $f_{O}(\underset{\sim}{v})$ is a linear function of $\underset{\sim}{v}$ close to the loss boundary, we find

$$
f_{e}^{(1)}=-e \phi(1) \exp (i k y-i \omega t) \frac{\partial f_{0}}{\partial \epsilon}
$$


at the unperturbed 1088 boundary. However, for $\omega \gg \nu_{e} f_{e}^{(1)}$ must fall almost to zero at a distance $\Delta v$ away from the loss boundary; see Fig. 2 . It follows that

$$
\frac{\partial f_{e}^{(1)}}{\partial v} \underset{\sim}{f_{e}^{(1)}} \simeq-\left(\frac{\omega}{\Delta v}\right)^{1 / 2}\left(\frac{m_{e}}{T_{e c}}\right)^{1 / 2} e \phi^{(1)} \frac{\partial f_{0}}{\partial \epsilon}
$$

near the loss boundary. For $\omega \ll \nu_{e}$. Eq. (30) is also satisfied at the unperturbed loss boundary, but now $f_{e}^{(1)}$ falls off on a scale (in velocity space) of the thermal velocity $\left(\mathrm{T}_{e c} / \mathrm{m}_{\theta}\right)^{1 / 2}$, so

$$
\frac{\partial f_{e}^{(1)}}{\partial \underset{\sim}{v}} \simeq\left(\frac{m_{e}}{T_{e c}}\right)^{1 / 2} \phi^{(1)} \frac{\partial f_{0}}{\partial \epsilon}
$$

Since the perturbed endloss current is proportional to $\partial f_{e}^{(1)} / \partial \underset{\sim}{\mathrm{v}}$ at the loss boundary. it follows from Eqs. (31) and (32) that the perturbed endloss current should be enhanced by a factor of order $\left(\omega / \nu_{e}\right)^{1 / 2}$ when $\omega>\nu_{e}$, i.e.

$$
\frac{4 \pi i}{\omega L} J_{\text {loss }}^{(1)} \simeq \frac{-i \omega_{\text {pec }}^{2} \nu_{\text {loss }}}{v_{e c}^{2}\left(\omega \nu_{e}\right)^{1 / 2}}
$$


A more rigorous calculation of $\mathrm{J}_{\text {loss }}(1)$ is done in the Appendix. We obtain (for $\omega \gg \nu_{e}$ )

$\frac{4 \pi i}{\omega L} J_{\text {loss }}^{(1)}=\frac{\exp (\pi i / 4) \omega_{\text {pec }}^{2} \nu_{\text {loss }}}{\left(2 \omega \nu_{e}\right)^{1 / 2} v_{e c}^{2}}\left(\frac{e \Phi}{T_{e c}}\right)^{1 / 4}\left[1+\left(\frac{R T e c}{e \Phi}\right)^{1 / 2}\right]_{\phi}^{-1}$

when $B_{\text {wall }} \ll B_{0}$ (appropriate for Tara). In Eq. (34). $R$ is the mirror ratio $B_{\max } / B_{0}$. We have assumed $\Theta \Phi \gg T_{e c}$, which is fairly well satisfied in Tara.

Using Eqs. (9), (13), (15), (17) and (34) we can now write the dispersion relation for low-frequency electrostatic flute modes in a square

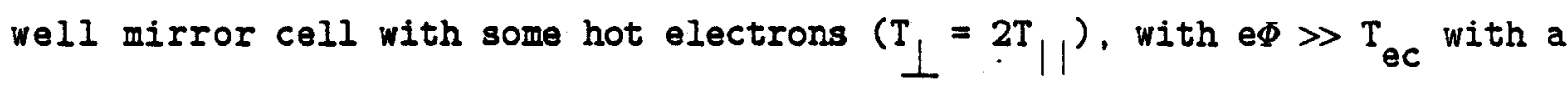
low magnetic field at cold end walls:

$$
\begin{aligned}
& \omega^{2}+\omega\left|\omega_{* i}\right|+\gamma_{\text {Ihd }, i}^{2}-\frac{\alpha \omega_{c i} \omega}{k_{R}}\left[1-\frac{\omega}{\pi_{\text {deh }}} \mathrm{Z}^{\prime}\left(\sqrt{\frac{\omega}{\omega_{\text {deh }}}}\right)\right] \\
& +\frac{\exp (\pi i / 4) \omega^{3 / 2} \bar{\nu}_{10 s s^{\omega}{ }^{\omega} \omega_{c e}}}{\sqrt{2} k^{2} v_{e c}^{2} \nu_{e}^{1 / 2}}\left(\frac{e \Phi}{T_{e c}}\right)^{1 / 4}\left[1+\left(\frac{R T}{e c}\right)^{1 / 2}\right]^{-1}=0
\end{aligned}
$$

where $\alpha \equiv n_{e h} /\left(n_{e h}+n_{e c}\right)$ is the fraction of hot electrons, and $\bar{\nu}_{\text {loss }}=(1-\alpha) \nu_{\text {loss }}$ is the mean loss rate for all electrons (hot and cold). 
Equation (35) is valid for $\omega \gg \nu_{\theta}, \omega \gg \hat{\omega}_{d i}$, and $k v_{i \perp} / \omega_{c i} \ll 1$. If $k v_{i \perp} / \omega_{c i}>1$, but still $\omega \gg \nu_{e}$, and $\omega \gg \hat{\omega}_{\mathrm{di}}$, we use Eq. (20) instead of Eq. (17) for the ion term, and obtain the dispersion relation

$$
\begin{aligned}
& \frac{(1-\alpha) \omega_{c i}{ }^{\omega}}{k R_{p}}+\frac{\alpha \omega_{c i}^{2} \omega_{c i}}{k R_{p}{ }^{\omega}{ }_{d e h}} Z^{\prime}\left(\sqrt{\frac{\omega}{\omega_{d e h}}}\right)+\frac{\omega^{2} \omega_{c i}}{k^{2} v_{i \perp}} 2\left[1-\left(1+\frac{\left|\omega_{* i}\right|}{\omega}\right) \exp \left(\frac{-k^{2} v_{i}^{2}}{\omega_{c i}^{2}}\right)\right. \\
& \left.\left.I_{0}\left(\frac{k^{2} v_{i}^{2}}{\omega_{c i^{2}}{ }^{2}}\right)\right]+\frac{\exp (\pi i / 4) \omega^{3 / 2} \nu_{10 s s}{ }^{\omega} c i^{\omega} c e}{\left(\frac{e \Phi}{T_{e c}}\right.}\right)^{1 / 4}\left[1+\left(\frac{\mathrm{RT} e c}{e \Phi}\right)^{1 / 2}\right]^{-1}=0
\end{aligned}
$$

$$
\text { If } \omega<\hat{\omega}_{\mathrm{di}} \text {, we must use the more general expression Eq. (11) for the }
$$
ion term, and obtain

$$
\begin{aligned}
& \frac{(1-\alpha) \omega_{c i}{ }^{\omega}}{k R_{p}}+\frac{\alpha \omega^{2} \omega_{c i}}{k R_{p} \omega_{d e, h}} z^{\prime}\left(\sqrt{\frac{\omega}{\omega_{d e h}}}\right)+\frac{\omega^{2} \omega_{c i}}{k^{2} v_{i \perp}^{2}}\left[1+\frac{\left(\omega\left|\omega_{* i}\right|\right.}{\omega_{d i}}\right)\left(\frac{\omega}{\omega_{d i}} \cdot \frac{k v_{i}}{\omega_{c i}}\right. \text {, } \\
& \left.\left.\frac{v_{i \perp}^{2}}{2 v_{i||}^{2}}\right)\right]+\frac{\exp (\pi i / 4) \omega^{3 / 2} \bar{\nu}_{l o s s} \omega_{c i} \omega_{c e}}{\sqrt{2 k^{2} v_{e c}^{2} \nu_{e}^{1 / 2}}}\left(\frac{e \phi}{T_{e c}}\right)^{1 / 4}\left[1+\left(\frac{R T e c}{e \phi}\right)^{1 / 2}\right]^{-1}=0
\end{aligned}
$$

where the function $G$ is defined by Eq. (10). 
If $\omega \ll \nu_{e}$ (but still $\omega \gg \nu_{\text {loss }}$ ), then the line-tying term in Eqs. (35) - (37) must be replaced by

$$
\frac{-4 \pi i \omega \omega_{c i}^{2}}{L k^{2} \omega_{p i}^{2}} \frac{\partial J_{l o s s}}{\partial \Phi} \simeq \frac{i \bar{\nu}_{l o s 8}{ }^{\omega \omega} c i{ }_{c e}}{k^{2} v_{e c}^{2}}\left(1-\frac{\omega_{* e c}}{\omega}\right)
$$

For cold ions and $\omega_{* e c} \ll \omega \ll \nu_{e}$, the dispersion relation is

$$
\omega^{2}-\frac{\alpha \omega_{c i}{ }^{\omega}}{k R_{p}}\left[1-\frac{\omega}{\hat{\omega}_{\mathrm{deh}}} \mathrm{Z}^{\prime}\left(\sqrt{\frac{\omega}{\hat{\omega}_{\mathrm{deh}}}}\right)\right]+\frac{i \bar{\nu}_{10 s s} \omega_{c i}{ }^{\omega} c e^{\omega}}{k^{2} v_{e c}^{2}}=0
$$

We can see the effect of the spread in hot electron drift velocities by comparing Eq. (39) to the dispersion relation that we would obtain if we had used a $\delta$ function distribution for the hot electrons

$$
f_{e h}\left(v_{\perp}, v_{\mid l}\right)=\left(2 \pi v_{0}\right)^{-1} \delta\left(v_{\mid l}\right) \delta\left(v_{\perp}-v_{0}\right)
$$

instead of Eq. (3). Then Eq. (5) would yield (for $k v_{0} / \omega_{c e} \ll 1$ )

$$
4 \pi \tilde{e}_{\text {eh }}^{(1)}=-\phi(1) \frac{\omega_{\text {peh }}^{2}}{v_{0}^{2}}\left[1-\frac{\omega-\omega_{* e h}}{\omega-\omega_{\mathrm{deh}}}\right]
$$




$$
\simeq-\phi(1) \frac{\omega_{p e h}^{2} k}{R_{p}\left(\omega-\omega_{d e h}\right) \omega_{c e}}
$$

The dispersion relation (for cold ions and $\omega \ll \nu_{e}$ ) would then be

$$
\left(\omega+\frac{i \vec{\nu}_{\text {loss }} \omega_{c i} \omega_{c \theta}}{k^{2} v_{\theta c}^{2}}\right)\left(\omega-\omega_{\text {deh }}\right)+\gamma_{\text {mhd,e }}^{2}=0
$$

where $\gamma_{\text {mhd.e }}^{2} \equiv \alpha\left(m_{e} / m_{i}\right)\left(v_{0}^{2} / 2 R_{p} R_{c}\right)$. If there were no line-tying. Eq. (42) would have an unstable solution

$$
\omega=\frac{\omega_{\mathrm{deh}}}{2}+i\left[\gamma_{\text {mhd }, e}-\frac{\omega_{\mathrm{deh}}^{2}}{4}\right]^{1 / 2}
$$

at $k<4 \gamma_{\text {mhd, }} e^{R_{c}{ }^{\omega}{ }_{c e} / v_{0}^{2}}$, which is the hot electron interchange instability. At $k \gg 4 \gamma_{\text {mhd, }} e_{c} c_{c e} / v_{0}^{2}$. Eq. (42) has the stable solutions

$$
\omega \simeq \omega_{\mathrm{deh}}
$$

$$
\text { and } \omega \simeq \gamma_{\text {mhd, }}^{2} / \omega_{\text {deh }}=\alpha \omega_{c i} / k R_{p}
$$


If we include a small line-tying term in Eq. (42), then we find that the unstable mode at $k<4 \gamma_{\text {mhd, }} e^{R} c \omega_{c e} / v_{0}^{2}$ is not affected much, but the stable precessional mode given by Eq. (44) acquires a small growth rate

$$
\omega \simeq \omega_{\text {deh }}+\frac{i \vec{\nu}_{10 s s^{\omega}}{ }_{c i}{ }^{\omega} c \gamma^{\gamma} \text { mhd,e }}{k^{2} v_{e c}^{2} \omega_{\text {deh }}^{2}}
$$

The line-tying, which acts like a positive dissipation, has de-stabilized the precessional mode, which is a negative energy wave. (The stable mode given by $\mathrm{Eq}$. (45) is a positive energy wave, and is slightly damped by the line-tying). If the line tying is made stronger, then the growth rate of the unstable MHD-like interchange mode at $k \ll 4 \gamma_{\text {mhd, }} e^{R} c{ }_{c e} / v_{0}^{2}$ is reduced to

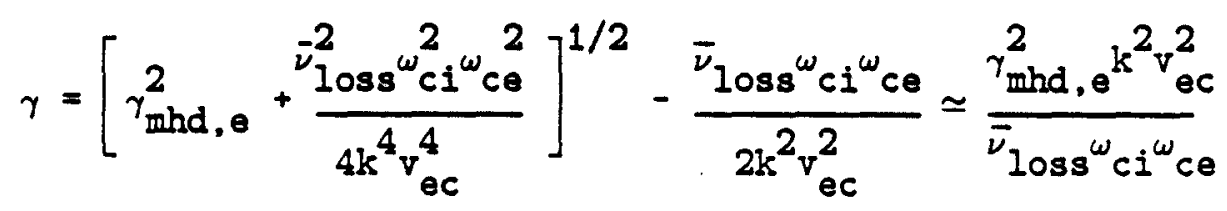

but the growth rate never goes to zero, and the modes at higher $k$ remain unstable. ${ }^{15}$ This precessional instability at high $\mathrm{k}$ is possible because the wave phase velocity is nearly equal to the drift velocity of the hot electrons, so that the wave can significantly perturb the hot electrons. This picture suggests that if the hot electrons had a spread in drift velocities, the precessional mode would be stabilized, since the wave phase 
velocity could not match all of the drift velocities. The MHD-like interchange mode, at low $k$, could also be stabilized if the line-tying term were to bring the growth rate $\gamma$ below $\hat{\omega}$ deh.

From Eq. (39), appropriate when the electrons have a bimaxwellian distribution and hence a spread in drift velocities, we find that, with no line-tying, we recover the MAD-like interchange instability with

$$
\omega \simeq i \gamma_{\text {mhd }, e} \equiv i\left(\frac{v_{e h}^{2}+v_{e h}^{2}}{2 R_{p} R_{c}}\right)^{1 / 2}
$$

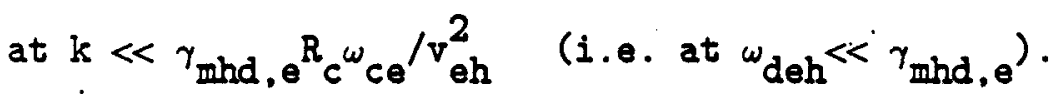

At $k \gg \gamma_{\text {mhd, }} e^{R} c{ }_{c e} / v_{e h}^{2}$, the growth rate is much lower, but the mode remains unstable, with

$$
\omega \simeq \frac{\alpha \omega_{c i}}{k R_{p}}\left(1+2 \sqrt{\pi i} \frac{\gamma_{\text {mhd,e }}^{3}}{\hat{\omega}_{\text {deh }}^{3}}\right)
$$

This unstable mode corresponds to the stable positive energy wave, given by Eq. (45), that we found for a $\delta$ function hot electron distribution. With a spread in drift velocities, this mode becomes unstable, because the resonant interraction with hot electrons drifting at the phase velocity provides a 
negative dissipation (i.e. a negative imaginary term in the dispersion relation from the imaginary part of $Z^{\prime}\left(\omega / \hat{\omega}_{\mathrm{deh}}\right)$.

So with either a spread in drift velocities alone (negative dissipation) or line-tying alone (positive dissipation), there are unstable modes at all $\mathrm{k}$. In contrast, when we have both line-tying and a spread in drift velocities, i.e. both positive and negative dissipative terms, then it is possible to achieve complete stabilization, rather than just a reduction in growth rate, because the positive dissipation can dominate for the positive energy wave, and the negative dissipation can dominate for the negative energy wave. 
III. Marginal Stability Condition

To show explicity that complete stabilization is possible, we now find the condition for marginal stability. For Tara parameters, the solutions to the dispersion relation $\mathrm{Eq}$. (35). divide neatly into hot electron driven modes (for which the ion drive term $\gamma_{\text {mhd,i }}^{2}$ is negligible) and ion driven modes (for which the ion inertia term $\omega^{2}$ is negligible, and the ion drive term is important). For the hot electron driven modes. Eq. (36) (which reduces to Eq. (35) without the $\gamma_{\text {mhd, } i}^{2}$ when $k v_{i \perp} / \omega_{c i} \ll 1$ ) is always a good approximation to the dispersion relation. Marginal stability of the hot electron driven mode will occur when the real and imaginary parts of Eq. (36) are both satisfied, for real w. (Note, however, that using Eq. (36) eliminates the ion driven modes, which may still be unstable when the hot electron driven modes are stable. The ion driven modes will be examined in Sec. IV). The real part of Eq. (36), for real $\omega$, is

$$
\begin{aligned}
& -\alpha \frac{{ }_{c i}{ }^{\omega}}{k R_{p}}\left[1-\frac{\omega}{\hat{\omega}_{\text {deh }}} \operatorname{Re} z^{\prime}\left(\sqrt{\frac{\omega}{\hat{\omega}_{\text {deh }}}}\right)\right]+\frac{{ }_{c i}^{\omega}}{k R_{p}} \\
& +\frac{\omega^{2} \omega_{c i}^{2}}{k^{2} v_{i \perp}^{2}}\left[1-\left(1+\frac{\left|\omega_{* i}\right|}{\omega}\right) \exp \left(\frac{-k^{2} v_{i \mid}^{2}}{\omega_{c i}^{2}}\right) I_{0}\left(\frac{k^{2} v_{i \perp}{ }^{2}}{\omega_{c i}^{2}}\right)\right]
\end{aligned}
$$


33

$$
+\frac{\omega^{3 / 2} \bar{\nu}_{\text {loss }} \omega_{c i}{ }^{\omega} c e}{2 k^{2} v_{e c}^{2} \nu_{e}^{1 / 2}}\left(\frac{e \Phi}{T_{e c}}\right)^{1 / 4}\left[1+\left(\frac{R T_{e c}}{e \Phi}\right)^{1 / 2}\right]^{-1}=0
$$

and the imaginary part of Eq. (36) is

$$
\begin{aligned}
& -2 \sqrt{\pi} \alpha \frac{\omega_{c i} \omega}{k R_{p}}\left(\frac{\omega}{\hat{\omega}_{\mathrm{deh}}}\right)^{3 / 2} \exp \left(\frac{-\omega}{\overline{\omega_{\mathrm{deh}}}}\right) \\
& +\frac{\omega^{3 / 2} \bar{\nu}_{10 s s^{\omega} c i^{\omega} c e}}{2 k^{2} v_{e c}^{2} \nu_{e}^{1 / 2}}\left(\frac{e \Phi}{T_{e c}}\right)^{1 / 4}\left[1+\left(\frac{R T}{e \Phi}\right)^{1 / 2}\right]^{-1}=0
\end{aligned}
$$

Eliminating $\alpha$ from Eggs. (50) and (51) yields an equation for $\omega$

$$
\begin{aligned}
& \frac{\omega_{c i}{ }^{\omega}}{k R_{p}}+\frac{\omega^{2} \omega_{c i}{ }^{2}}{k^{2} v_{i}^{2}}\left[1-\left(1+\frac{\left|\omega_{* i}\right|}{\omega}\right) \exp \left(\frac{-k^{2} v_{i \perp}{ }^{2}}{\omega_{c i}{ }^{2}}\right) I_{0}\left(\frac{k^{2} v_{i}^{2}}{\omega_{c i}{ }^{2}}\right)\right] \\
& +\frac{\bar{\nu}_{10 s s^{\omega}{ }^{\omega}{ }^{\omega} c \theta}}{2 k^{2} v_{e c}^{2} \nu_{e}^{1 / 2}}\left(\frac{e \Phi}{T_{e c}}\right)^{1 / 4}\left[1+\left(\frac{R T}{e \Phi}\right)^{1 / 2}\right]-1 \mid \omega^{3 / 2} \\
& \left.-\frac{\hat{\omega}_{\mathrm{deh}}^{3 / 2}}{2 \pi^{1 / 2}} \exp \left(\frac{\omega}{\hat{\omega}_{\mathrm{deh}}}\right)\left[1-\frac{\omega}{\hbar_{\mathrm{deh}}} \operatorname{Re} z\left(\sqrt{\frac{\omega}{\hat{\omega}_{\mathrm{deh}}}}\right)\right]\right\}=0
\end{aligned}
$$


Equation (52) may be solved for $w$ numerically or (in certain limits) analytically, and the result put into Eq. (51) to find the value of the hot electron fraction $\alpha$ which will make the hot electron driven interchange mode marginally stable, for a given $k$. For $\alpha$ above this value, the mode will be unstable, while for $\alpha$ below this value, it will be damped. We will first give some analytic results for the marginally stable $\alpha(k)$, and show that the assumptions we made (that the ion drive term is negligible, that $\omega>\nu_{e}$. and that $\omega \gg \omega_{d i}$ ) are justified for Tara parameters. We will then show the results of some numerical calculations of the marginally stable $\alpha(k)$, and discuss the relation between these results and the observations of hot electron interchange modes in Tara.

For $k v_{i \perp} / \omega_{c i} \ll 1$. Eq. (52) reduces to

$$
\begin{aligned}
& \omega^{2}+\omega\left|\omega_{* i}\right|+\frac{\bar{\nu}_{10 s s_{c i}^{\omega} \omega_{c e}}}{2 k^{2} v_{e c} \nu_{e}^{1 / 2}}\left(\frac{e \Phi}{T_{e c}}\right)^{1 / 4}\left[1+\left(\frac{R T_{e c}}{e \Phi}\right)^{1 / 2}\right]^{-1}\left\{\omega^{3 / 2}\right. \\
& \left.-\frac{\hat{\omega}_{\text {deh }}^{3 / 2}}{2 \pi^{1 / 2}} \exp \left(\frac{\omega}{\hat{\omega}_{\text {deh }}}\right)\left[1-\frac{\omega}{\hat{\omega}_{\text {deh }}} \operatorname{Re} z^{\prime}\left(\sqrt{\frac{\omega}{\hat{\omega}_{\text {deh }}}}\right)\right]\right\}=0
\end{aligned}
$$

For $k$ sufficiently small, the first two terms of Eq. (53) can be neglected, and Eq. (53) reduces to 


$$
\omega^{3 / 2}-\frac{\hat{A}_{\mathrm{deh}}^{3 / 2}}{2 \pi^{1 / 2}} \exp \left(\frac{\omega}{\hbar_{\mathrm{deh}}}\right)\left[1-\frac{\omega}{\pi_{\mathrm{deh}}} \operatorname{Re} Z^{\prime}\left(\sqrt{\frac{\omega}{\hbar_{\mathrm{deh}}}}\right)\right]=0
$$

which has the solution $\omega=0.7 \hat{\omega}_{\text {deh }}$. Then, from Eq. (51), marginal stability occurs when

$$
\alpha \simeq 0.23 \frac{\bar{\nu}_{10 s s_{c e}^{\omega}{ }^{1 / 2} v_{e h}^{R} p}}{\nu_{e}^{1 / 2} v_{e c}^{2} R_{c}^{1 / 2}{ }_{k}^{1 / 2}}\left(\frac{e \Phi}{T_{e c}}\right)^{1 / 4}\left[1+\left(\frac{R T e c}{e \Phi}\right)^{1 / 2}\right]^{-1}
$$

where we have made use of the definition of $\hat{\omega}_{\text {deh }}$, following Eq. (11). In this regime, where line-tying plays a dominant role, the modes at low $k$ are more easily stabilized (i.e. they require a higher hot electron fraction $\alpha$ to be unstable) than modes at higher $k$. Note that in the Tara plug. $\mathrm{T}_{\mathrm{eh}} \simeq$ $200 \mathrm{keV}, \mathrm{R}_{\mathrm{p}} \simeq 10 \mathrm{~cm}, \mathrm{~B}_{0} \simeq 4 \mathrm{kG}, \mathrm{R}_{\mathrm{c}} \simeq 10^{3} \mathrm{~cm}$, and in the central cell (which dominates the Iine-tying) $\mathrm{T}_{\mathrm{ec}} \simeq 40 \mathrm{eV}$, and $\mathrm{n}_{\mathrm{ec}} \simeq 2 \times 10^{11} \mathrm{~cm}^{-3}$, so $\omega_{\mathrm{deh}} \simeq 2$ $\times 10^{5} \mathrm{sec}^{-1}$ for $\mathrm{m}=1$ (i.e. $\mathrm{kR}_{\mathrm{p}}=1$ ) and proportionally higher for higher $\mathrm{m}$, while $\nu_{e} \simeq 2 \times 10^{4} \mathrm{sec}^{-1}$. Hence we are justified in assuming $\omega>\nu_{e}$ for all modes in this regime. 
This regime breaks down when $k$ is sufficiently large that either the $\omega^{2}$ term or the $\omega\left|\omega_{* 1}\right|$ term in Eq. (53) becomes comparable to the last two terms. For cold ions, $\omega\left|\omega_{* i}\right|$ is always zero, so Eq. (55) stops being valid when $\omega^{1 / 2} \simeq \hat{\omega}_{\text {deh }}^{1 / 2}>\bar{\nu}_{\text {loss }} \omega_{c i} \omega_{c e} / k^{2} v_{\theta c}^{2} \nu_{e}^{1 / 2}$, or

$$
k>\frac{\nu_{10 s s}^{2 / 5} \omega_{c i} R_{c}^{1 / 5}}{v_{e h}^{2 / 5} v_{e c}^{4 / 5} \nu_{e}^{1 / 5}}\left(\frac{m_{i}}{m_{e}}\right)^{3 / 5}
$$

Above this value of $k, \omega \ll \hat{\omega}_{\text {deh, }}$ and Eq. (53) reduces to

$$
\omega^{2} \cdot \frac{\bar{\nu}_{10 s s^{\omega} i_{c e} \omega_{c e h} \hat{\omega}_{e j}^{3 / 2}}}{4 \pi^{1 / 2} \mathrm{k}^{2} v_{e c}^{2} \nu_{e}^{1 / 2}}\left(\frac{\mathrm{e} \Phi}{\mathrm{T}_{e c}}\right)^{1 / 4}\left[1+\left(\frac{\mathrm{RT}}{\mathrm{ec}}\right)^{1 / 2}\right]^{-1}=0
$$

and Eq. (51) yields

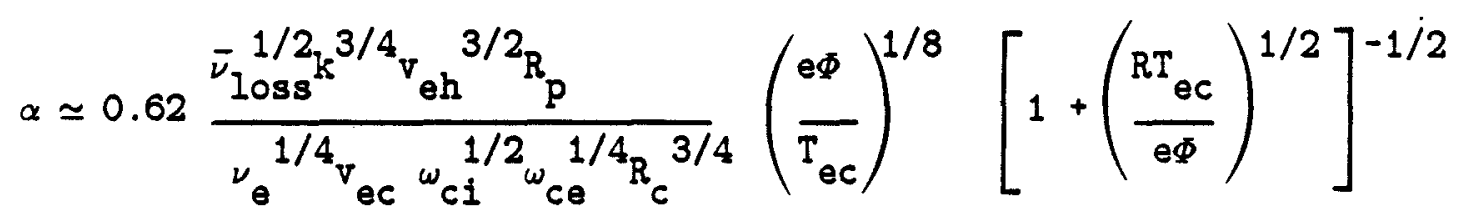

In this regime, modes at higher $k$ are more easily stabilized than modes at lower $k$, because line-tying (which is stronger at low $k$ ) no longer dominates the dispersion relation, and the higher $k$ modes are more weakly coupled to hot electrons. It follows that the most difficult mode to 
stabilize (the first mode to go unstable as $\alpha$ increases) will be at roughly the intermediate value of $\mathrm{k}$ between the two regimes, given by Eq. (56), i.e.

$k_{c r i t} \simeq 0.77 \frac{\bar{\nu}_{\text {loss }}^{2 / 5} \omega_{c i} R_{c}^{1 / 5}}{v_{e h}^{2 / 5} v_{e c}^{4 / 5}{ }_{\nu_{e}}^{1 / 5}}\left(\frac{m_{i}}{m_{e}}\right)^{3 / 5}\left(\frac{e \Phi}{T_{e c}}\right)^{1 / 10}\left[1+\left(\frac{\mathrm{RT}_{e c}}{e \Phi}\right)^{1 / 2}\right]^{-2 / 5}$

and this mode will first go unstable at

$\alpha_{\text {crit }} \simeq 0.30 \frac{\bar{\nu}_{10 s v_{e h}^{6 / 5} R_{p}}^{4 / 5}}{\nu_{e}^{2 / 5}{ }_{R_{c}}^{3 / 5} v_{e c}^{8 / 5}}\left(\frac{m_{i}}{m_{e}}\right)^{1 / 5}\left(\frac{e \Phi}{T_{e c}}\right)^{1 / 5}\left[1+\left(\frac{R T e c}{e \Phi}\right)^{1 / 2}\right]^{-4 / 5}$

which is the point where Eq. (58) crosses Eq. (55). This result is only valid if the ions are cold enough so that $\left|\omega_{* i}\right|<\omega$ at $k=k_{\text {crit }}$. This is true if

$$
T_{i}<T_{e h} R_{p} / R_{c}
$$

or $\mathrm{T}_{i}<1 \mathrm{keV}$ in Tara. If $\mathrm{T}_{i}$ is higher than this, then instead of $\mathrm{Eq}$. (57), we have 


$$
\omega\left|\omega_{* i}\right|-\frac{\bar{\nu}_{\mathrm{Ioss}} \omega_{c i}{ }^{\omega} \mathrm{ce}^{\omega}{ }_{\mathrm{deh}}^{3 / 2}}{4 \pi^{1 / 2} k^{2} v_{e c}^{2} \nu_{e}^{1 / 2}}\left(\frac{e \Phi}{T_{e c}}\right)^{1 / 4}\left[1+\left(\frac{\mathrm{RT}}{e \Phi}\right)^{1 / 2}\right]^{-1}=0
$$

when

$$
k>\frac{\bar{\nu}_{\text {loss }}^{2 / 5} \omega_{c i}^{4 / 5} \omega_{c e}^{1 / 5} R_{p}^{2 / 5} v_{e h}^{2 / 5}}{v_{i}^{4 / 5} v_{e c}^{4 / 5} R_{c}^{1 / 5} \nu_{e}^{1 / 5}}
$$

Then Eq. (52) yields

$$
\alpha \simeq \frac{k^{2}\left(v_{i}{ }^{2}+v_{i}^{2}\right)}{2 \omega_{c i}^{2}}
$$

for marginal stability (valid for $\alpha \ll 1$ ). In this regime, higher $k$ modes are more easily stabilized, so again the most difficult mode to stabilize occurs at the boundary between this regime and the low $\mathrm{k}$ regime where Eq. (55) is valid, i.e.

$k_{\text {crit }} \simeq 0.39 \frac{\bar{\nu}_{\text {loss }}^{2 / 5} \omega_{c i}^{4 / 5} \omega_{c e}^{1 / 5} R_{p}^{2 / 5} v_{e h}^{2 / 5}}{v_{i}^{4 / 5} v_{e c}^{4 / 5} R_{c}^{1 / 5}{ }_{\nu}^{1 / 5}}\left(\frac{e \Phi}{T_{e c}}\right)^{1 / 10}\left[1+\left(\frac{R_{e c}}{e \Phi}\right)^{1 / 2}\right]^{-2 / 5}$ 
and

$\alpha_{\text {crit }} \simeq 0.43 \frac{\nu_{\text {loss }}^{4 / 5} v_{i}^{2 / 5} R_{p}^{4 / 5} v_{e h}^{4 / 5}}{\nu_{\theta}^{2 / 5} v_{e c}^{8 / 5} R_{c}^{2 / 5}}\left(\frac{m_{i}}{m_{\theta}}\right)^{2 / 5}\left(\frac{e \Phi}{T_{e c}}\right)^{1 / 5}\left[1+\left(\frac{R T_{e c}}{e \Phi}\right)^{1 / 2}\right]^{-4 / 5}$

It may be seen from Eq. (64) that if $\alpha_{\text {crit }}$ (given by Eq. (66)) is much less than 1, then $k v_{i \perp} / \omega_{c i} \ll 1$ for $k<k_{c r i t}$, and we were justified in assuming $\mathrm{kv}_{i \perp} / \omega_{c i} \ll 1$ in deriving Eq. (53) from Eq. (52). If $\gamma_{\text {mhd, } i}^{2} \ll \omega\left|\omega_{* i}\right|$ for $k$ $\simeq k_{\text {crit }}$ (given by Eq. (66)), then we were justified in neglecting the ion drive term in Eq. (54). The condition for this is

$$
T_{i} \ll \frac{m_{i}^{1 / 4} T_{e h}^{7 / 4} \nu_{10 s s^{R} p}}{T_{e c} R_{c}^{1 / 2} \nu_{e}^{1 / 2}}
$$

In steady state operation, the loss rate $\nu_{\text {loss }}$ must be equal to the ionization rate $n_{0}<\sigma v>$, where $n_{0}$ is the neutral density and $<\sigma v>$ is the electron ionization cross-section for atomic hydrogen (since most hydrogen molecules are dissociated before penetrating very far into the plasma) averaged over the electron distribution. (In fact the hot electron interchange mode is seen in Tara after the ECRH is turned off, when the central cell electron density is falling at a rate comparable to $n_{0}\langle\sigma v\rangle$, so 
$\nu_{\text {loss }}$ may be somewhat greater than $\mathrm{n}_{0}\langle\sigma \mathrm{v}\rangle$.) In Tara, $\left.\mathrm{n}_{0}<\sigma \mathrm{v}\right\rangle$ is typically around $10^{3} \sec ^{-1}$ (for $n_{0}=10^{11} \mathrm{~cm}^{-3}$, corresponding to a neutral gas pressure of $3 \times 10^{-6}$ torr). In this case. Eq. (67) becomes $T_{i} \ll 100 \mathrm{keV}$, which is satisfied for Tara. At the lowest gas pressures, and at the highest ion temperatures that are expected when neutral beams are used in the plug $\left(T_{i}=10 \mathrm{keV}\right)$. Eq. (67) way be only marginally satisfied. In this case, there is no longer a clear cut distinction between hot electron driven modes and ion driven modes, and it is necessary to solve the full dispersion relation. Eq. (37), without making any approximations (except for $\omega \gg \nu_{e}$ ).

Marginal stability curves $\alpha(k)$ have been found numerically from Eqs. (51) and (52). In all cases we assumed $\mathrm{T}_{\perp}=2 \mathrm{~T}_{\|}$for the hot electrons and the ions (so $T \equiv \frac{2}{3} T_{\perp}+\frac{1}{3} T_{\|}=\frac{5}{3} T_{\|}$), and used $T_{e h}=200 \mathrm{keV}, T_{e c}=40 \mathrm{eV}$. $R_{p}=10 \mathrm{~cm}, R_{c}=10 \mathrm{~m}, \Phi=100 \mathrm{~V}$, mirror ratio $R=6$, and $B_{0}=4 \mathrm{kG}$. The cold electron density (needed to find $\nu_{e}$ ) was held fixed at $1.5 \times 10^{11} \mathrm{~cm}^{-3}$, and the loss rate $\nu_{\text {loss }}$ was held fixed at $n_{0}<\sigma v>$ with $<\sigma v>=10^{-8} \mathrm{~cm}^{3} \mathrm{sec}^{-1}$. independent of $\alpha$, in order to make Eq. (53) independent of $\alpha$. (Actually $\mathrm{n}_{e c}$ and $<\sigma r>$ should depend weakly on $\alpha$, and an iterative process should be used to solve Eqs. (51) and (52) for $\alpha$ and $\omega$ ). The results are shown in Fig. 3 (for $n_{0}=3 \times 10^{11} \mathrm{~cm}^{-3}$. corresponding to a neutral gas pressure of 
$10^{-5}$ torr) and Fig. 4 (for $n_{0}=3 \times 10^{10} \mathrm{~cm}^{-3}$, corresponding to $10^{-6}$ torr). The scale on the horizontal axis gives $k R_{p}$. which corresponds to the azimuthal mode number $m$ in a cylindrical plasma. In each figure, marginal stability curves are shown for three different ion temperatures. $T_{i}=100$ eV, $1 \mathrm{keV}$ and $10 \mathrm{keV}$. As expected, higher $\mathrm{n}_{0}$ (which means a higher end loss current, and stronger line-tying) results in greater stability, i.e. in a higher hot electron fraction $\alpha$ needed for instability. At $T_{i}=100 \mathrm{eV}$, the critical $\alpha$ (the lowest $\alpha$ at which any $k$ is unstable) is $\alpha_{\text {crit }}=12 \%$ for $n_{0}=$ $3 \times 10^{11} \mathrm{~cm}^{-3}$, while $\alpha_{\text {crit }}=2 \%$ for $\mathrm{n}_{0}=3 \times 10^{10} \mathrm{~cm}^{-3}$. The first mode to go unstable when $\alpha$ reaches $\alpha_{\text {crit }}$ is $\mathrm{m}_{\text {crit }}=5$ for $\mathrm{n}_{0}=3 \times 10^{11} \mathrm{~cm}^{-3}$ and $\mathrm{m}_{\text {crit }}$ $=2$ for $n_{0}=3 \times 10^{10} \mathrm{~cm}^{-3}$. As expected, increased line-tying causes an increase in $\mathrm{m}_{\text {crit. }}$, since it is the low m modes that are most effectively stabilized by line-tying. These numerical results are comparable to the approximate analytic results (which neglected factors of order unity) for the cold ion regime, given by Eqs. (59) and (60) (where $k_{\text {crit }}$ is equivalent to $m_{c r i t}\left(R_{p}\right)$. As we also expected, increasing the ion temperature results

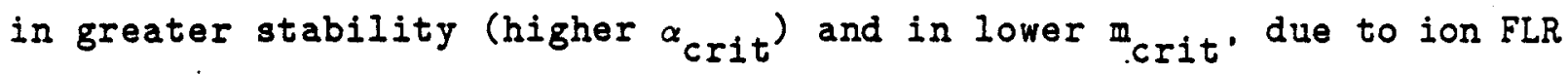
(which is more effective at stabilizing high m). At $T_{i}=10 \mathrm{keV}$, for $\mathrm{n}_{0}=3$ $\times 10^{11} \mathrm{~cm}^{-3}, \alpha_{\text {crit }}=22 \%$ and $m_{\text {crit }}=1$, while for $n_{0}=3 \times 10^{10} \mathrm{~cm}^{-3}, \alpha_{c r i t}=$ 
$4 \%$ and $m_{\text {crit }}=0.5$ (or, since $m=0.5$ is not possible, $\alpha=6 \%$ at $m=1$ ). These results are comparable to the estimates given by Eqs. (65) and (66). which should be valid for $T_{i}>T_{e h} R_{p} / R_{c} \simeq 1 \mathrm{keV}$. However (as we would expect from the analytic results), raising $T_{i}$ has a fairly weak stabilizing effect. From Eq. (66), $\alpha_{\text {crit }}$ scales only as $T_{i}^{1 / 5}$ when $T_{i} \gg T_{e h} R_{p} / R_{c}$, and from Eq. (60), $\alpha_{c r i t}$ is independent of $T_{i}$ for $T_{i} \ll T_{e h} R_{p} / R_{c}$. Marginal stability curves have also been calculated from Eq. (37), which includes the ion drive term and does not assume $\omega \gg \omega_{\mathrm{di}}$. The results for $\alpha_{c r i t}$ and $m_{\text {crit }}$ are nearly the same (although there are some differences at $m>$ $\left.m_{\text {crit }}\right)$, except for $n_{0}=3 \times 10^{10} \mathrm{~cm}^{-3}$ at $T_{i}=10 \mathrm{keV}$, for which we were unable to find any marginally stable modes. In this case Eq. (67) is not satisfied, so there is no clean distinction between hot electron driven modes and ion driven modes.

A direct comparison between these results and the observations of hot electron interchange modes in Tara is possible only if the modes in Tara are electrostatic and flutelike, extending throughout the length of the machine. In this case, the hot electron fraction $\alpha$ should be interpreted as $\mathrm{n}_{\mathrm{eh}} \mathrm{V}_{\mathrm{p}} /$ $\left(n_{p} V_{p}+n_{c c} V_{c c}\right)$, where $n_{e h}$ is the hot electron density in the plug, $n_{p}$ and $\mathrm{n}_{c c}$ are the total electron densities in the plug and in the central cell, and $V_{p}$ and $V_{c c}$ are the volumes of the plug and central cell. Since $n_{p} V_{p} \ll$ 
$n_{c c} V_{c c}$, the effective $\alpha$ is approximately $n_{e h} V_{p} / n_{c c} V_{c c}$. As $n_{c c}$ drops (but $n_{\text {eh }}$ remains almost constant) after the ECRH is turned off, the effective $\alpha$ will increase, and we would expect the instability to appear (at m = m $c$ rit) when $\alpha$ reaches $\alpha_{c r i t}$ i.e. when $n_{c c}$ reaches $\left(n_{e h} / \alpha_{c r i t}\right)\left(v_{p} / v_{c c}\right)$. For $n_{e h}=$ $5 \times 10^{10} \mathrm{~cm}^{-3}, v_{c c} / v_{p}=20$, and $\alpha_{c r i t}=0.12$ (corresponding to $n_{0}=3 \times 10^{11}$ $\mathrm{cm}^{-3}, \mathrm{I}_{i}=100 \mathrm{eV}$, the lower curve in Fig. 3), we expect the $\mathrm{m}=5$ mode to go unstable first, at $\mathrm{n}_{c c}=2 \times 10^{10} \mathrm{~cm}^{-3}$. In fact, for $\mathrm{n}_{0}=3 \times 10^{11} \mathrm{~cm}^{-3}$. $T_{i}=100 \mathrm{eV}$, the instability typically has $\mathrm{m} \simeq 9$ to 15 , in fair agreement with the predicted $\mathrm{m}_{c r i t}=5$, but it first appears at $\mathrm{n}_{c c} \simeq 2.5 \times 10^{11} \mathrm{~cm}^{-3}$. about 10 times higher than our model predicts. This discrepancy is most likely due to the modes in Tara not being very flutelike. Although a detailed calculation of the axial mode structure is still in progress and will be reported in a future paper, we note that the modes will be flutelike if there are enough electrons passing between the center cell and the plug. In particular,the fraction of passing electrons in the central cell. $n_{\text {pass }} / n_{c c}$ must satisfy

$$
n_{p a s s} L_{p} / n_{c c} L_{c c}>k^{2} T_{e c} / m_{i} \omega_{c i}{ }^{2}
$$

and

$$
\left.n_{\text {pass }} L_{p} / \Omega_{c c} L_{c c} \gg \nu_{\text {loss }} /(\omega \nu)\right)^{1 / 2}
$$


Equation (68) is the usual condition for trapped particle modes to be flutelike ${ }^{2}$ (and hence not to have MHD-like growth rates); Eq. (69) is an analogous condition that must also be satisfied when line-tying (with $\omega$ > $\left.\nu_{e}\right)$ is included in the dispersion relation for trapped particle modes. In Tara, $n_{\text {pass }} L_{p} / n_{c c} L_{c c}$ is about $2 \times 10^{-3}$. For $m=10$ and $\omega=2 \pi \times 10^{6}$ $\sec ^{-1}$ (a typical mode number and frequency observed in Tara), and $\nu_{\text {loss }}=3$ $\times 10^{3} \mathrm{sec}^{-1}$ (appropriate for $\mathrm{n}_{0}=3 \times 10^{11} \mathrm{~cm}^{-3}$ ), we find $\mathrm{k}^{2} \mathrm{~T}_{e c} / \mathrm{m}_{i} \omega_{c i}{ }^{2} \simeq$ $10^{-2}$ and $\nu_{\text {loss }} /\left(\omega \nu_{e}\right)^{1 / 2} \simeq 2 \times 10^{-2}$. Since Eq. (68) and (69) fail to be satisfied by a factor of 5 or 10, we expect the modes to be localized in the plug, but to still have a substantial amplitude in the central cell, perhaps $10 \%$ or $20 \%$ of their amplitude in the plug. (Probes in the central cell of Tara see RF bursts at the same time as the bursts are seen in the plug, so there is evidence that the mode amplitude is not zero in the central cell. but it has not yet been possible to measure the relative amplitude in the central cell and plug. There is also evidence ${ }^{6}$ that there is some non-zero mode amplitude in the anchor, but less than in the plug, since turning on the anchor reduces the central cell electron density needed for stability.) The line-tying term will be reduced by $\bar{\phi}^{(1)} / \phi_{\mathrm{p}}^{(1)}$, where $\bar{\phi}^{(1)}$ is the average perturbed potential seen by electrons whose turning points are near the end walls, and $\phi_{\mathrm{p}}^{(1)}$ is the perturbed potential in the plug. Furthermore, the cold electrons in the central cell, where the mode amplitude is lower, will 
not be as effective at stabilizing the mode as they would be if the mode were flutelike, so the effective $\alpha$ will be greater than $n_{e h} V_{p} / n_{c c} V_{c c}$. So it is not surprising that the instability occurs when $\mathrm{n}_{\mathrm{cc}} \simeq 2.5 \times 10^{11} \mathrm{~cm}^{-3}$. about 10 times higher than our model would predict if the modes were flutelike.

A more direct comparison may be made between our model and the results of a single cell mirror experiment by Brown et.al. ${ }^{27}$, who observed hot electron interchange modes that presumably were flute-like. In that experiment, hot electrons were produced by short pulse ECR heating of a lithium plasma (created by contact ionization) in a background of a neutral noble gas, at a magnetic fleld of 3kG. Typical hot electron parameters after heating were $\mathrm{n}_{e h}=10^{12} \mathrm{~cm}^{-3}, \mathrm{~T}_{\text {eh }}=10 \mathrm{keV}$, and $\mathrm{R}_{\mathrm{p}}=0.5 \mathrm{~cm}$. The magnetic mirrors were $15 \mathrm{~cm}$ apart in a $25 \mathrm{~cm}$ r.f. cavity which terminated the plasma. A mirror ratio $\mathrm{R}=1.5$ created an unfavorable curvature of $R_{c}=50 \mathrm{~cm}$. The hot electrons drove an unstable interchange mode which caused a sudden drop in plasma density a few growth periods after the ECRH pulse; while the mode was growing, background neutrals were ionized, producing a substantial cold electron population with $\mathrm{T}_{\mathrm{ec}} \simeq 10 \mathrm{eV}$. The mode was stabilized, and the density stopped falling, when the hot electron fraction a fell to a certain level. In a typical run, using krypton at $5 \times 10^{-5}$ torr as the background gas, the loss rate $\nu_{\text {Ioss }}$ was measured to be 
$9 \times 10^{3} \mathrm{sec}^{-1}$ after the plasma stabilized, and $\alpha$ was found to be 0.17 . There is some evidence that most of the lithium ions were lost in the dump of plasma associated with the instability, and that when stability was reached, most of the ions were krypton. But there may also have been some lithium ions present, and some uniknown fraction of krypton ions that were doubly ionized. If there are several ion species present with different mass $m_{i}$ and charge $q_{i}$, then $m_{i} / q_{i}$ should be replaced by $\left\langle m_{i} / q_{i}\right\rangle$ in the dispersion relations, Eqs. (35) - (37), and in the expressions for the marginally stable $\alpha$ and $k_{\text {crit }}$ If the composition is $50 \% \mathrm{Kr}^{+}, 25 \% \mathrm{Li}^{+}$, and $25 \% \mathrm{Kr}^{++}$, then $\left\langle\mathrm{m}_{i} / q_{i}\right\rangle \simeq 60 \mathrm{~m}_{H} / \theta$, where $\mathrm{m}_{\mathrm{H}}$ is the mass of a proton. Using this figure to find $\omega_{c i}$ and $m_{i} / m_{\theta}$, and using Eq. (23) to find $\nu_{e}$ for the cold electrons at $\mathrm{n}_{\mathrm{ec}}=5 \times 10^{12} \mathrm{~cm}^{-3}$, and using $e \Phi / \mathrm{T}_{\mathrm{ec}} \simeq \ln \left(\nu_{e} / \nu_{\text {loss }}\right) \simeq 6$, we find from Eq. (59) that $k_{\text {crit }} R_{p} \simeq 4 \times 10^{-2}$. Since the smallest possible $\mathrm{k}$ (the $\mathrm{m}=1$

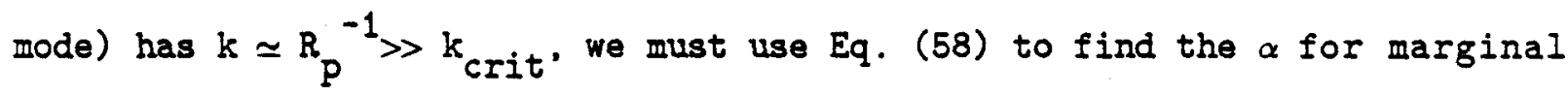
stability, and we obtain $\alpha=0.30$, which is within a factor of 2 of the observed value, $\alpha=0.17$. For a pure $\mathrm{Li}^{+}$plasma, we would obtain $\alpha=0.10$. The theory of Berk ${ }^{19}$, which uses a monoenergetic hot electron distribution and ignores line-tying, and which Brown et.al. used to explain their results, gives $\alpha=4$ for the same mixture of ion species, (taking $T_{\text {eh }} \mid=0$ and $\mathrm{T}_{\text {eh }}=10 \mathrm{keV}$ ) and $\alpha=0.5$ even for pure $\mathrm{Li}^{+}$, in much worse agreement 
with the experiment. At higher gas pressures, up to $2 \times 10^{-4}$ torr, the measured $\nu_{\text {loss }}$ increases, so we would expect from Eq. (58) that $\alpha$ would increase, but the experimentally observed a remains nearly the same. However, there is reason to believe that some anomalous loss process, other than classical end losses, may be operating at these higher pressures, and the anomalous loss rate could have a different dependence on the potential $\Phi$ than classical end losses, so our line-tying model would not be applicable. The results using gases other than krypton seem not to scale with $m_{i}$ as one would expect from Eq. (58), but the marginally stable $\alpha$ was measured by a different, more indirect method in these cases, and the interpretation of these results is uncertain. This indirect method was also used to measure the dependence of the marginally stable $\alpha$ on $\mathrm{T}_{\mathrm{eh}}$, and it was stated in Brown et.al. 27 that $\alpha$ goes as $\mathrm{T}_{\mathrm{eh}}{ }^{1.7}$, while $\mathrm{Eq}$. (58) would predict that $\alpha$ would go as $\mathrm{T}_{e}^{3 / 4}$. Because of the indirect method used, and the spread in data points, a could go as $\mathrm{T}_{\mathrm{eh}}{ }^{3 / 4}$ within the range of uncertainty of the experiment. 28 
IV. Ion Driven Modes.

In addition to the hot electron driven interchange modes discussed in Sec. II, the dispersion relation Eq. (37) also describes ion driven interchange modes. (Equation (36), which was the basis for the results given in Sec. III, does not include ion driven modes, because it does not have an ion drive term). If $\omega \gg \hat{\omega}_{\mathrm{di}}$, then $\mathrm{Eq}$. (35) may be used. Because $\omega$ $\ll \hat{\omega}_{\text {deh }}$ for these modes, the negative dissipation from the hot electrons will always be negligible compared to the positive dissipation from the line-tying, and ( if $\omega \gg \hat{\omega}_{\mathrm{di}}$ ) the ion driven modes will always be unstable. If $\omega<\hat{\omega}_{\mathrm{di}}$. (In which case Eq. (37) must be used), then the ion term will have a resonant part which acts like a negative dissipation, and the ion driven modes may be stable; this can be true for all $k$ only if $T_{i}$ is rather high (tens of keV, for Tara parameters). Both Eq. (35) and Eq. (37) assume $\omega \gg \nu_{e}$; if this is not true. Eq. (38) must be substituted for the linetying term in Eq. (35) or Eq. (37). For Tara parameters we find $\omega>\nu_{e}$ for the ion driven modes, except for the lowest mode numbers $m$ at low $T_{i}$ (less than a few hundred eV).

Using Eq. (35) with $\omega \ll \hat{\omega}_{\text {deh }}$, the dispersion relation is 


$$
\begin{aligned}
& \omega^{2}+\dot{\omega}\left|\omega_{* i}\right|-\frac{\alpha_{c i} \omega}{k R_{p}}+\gamma_{\text {mhd }, i}^{2} \\
& \left.+\frac{\exp (\pi i / 4) \omega^{3 / 2} \bar{\nu}_{\text {loss }{ }^{\omega} c i^{\omega} c \theta}}{\sqrt{2} k^{2} v_{e c}^{2} \nu_{e}^{1 / 2}}\right)^{1 / 4}\left[1+\left(\frac{R T}{e c}\right)^{1 / 2}\right]^{-1}=0
\end{aligned}
$$

At low $\vec{\nu}_{\text {loss }}$ and low ion temperature

$$
T_{i} \ll \alpha^{4 / 3} \nu_{e}^{2 / 3} T_{e c} \gamma_{\text {mhd, }, \theta}^{2 / 3} \bar{\nu}_{\text {loss }}^{-4 / 3}
$$

or $\mathrm{T}_{i}<100^{\circ} \mathrm{eV}$ for Tara parameters, the line-tying term in Eq. (70) is always small. Then we find that at $k v_{11} / \omega_{c i}=\alpha^{1 / 2}$, the ion FLR term $\omega\left|\omega_{* i}\right|$ and the hot electron term $\alpha \omega_{c i} \omega / k R_{p}$ cancel, and $\omega=i \gamma_{\text {mhd, } i}$. (This result assumes $\alpha<1$, otherwise $\mathrm{kv}_{i \perp} / \omega_{c i}>1$ and $\mathrm{Eq}_{\mathrm{q}}$ (70) will not be valid). This MHD growth rate only occurs over a narrow range of $\mathrm{k}$, viz. $\mid \mathrm{kv}_{i \perp} / \omega_{c i}$ $\alpha^{1 / 2} \mid<\left(R_{p} / R_{c}\right)^{1 / 2}$. Well outside this range of $k$, the mode is nearly stable, with a small growth rate due to the line-tying term 


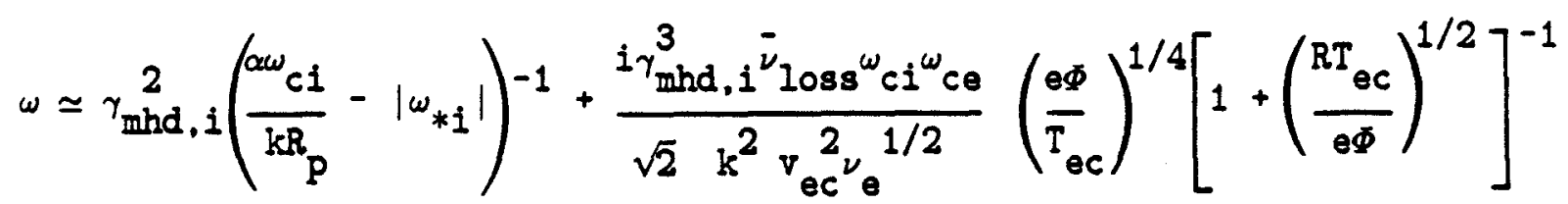

$$
\left|\frac{\alpha \omega_{c i}}{k R_{p}}-\right| \omega_{* i}||^{-5 / 2} .
$$

At higher ion temperature, the line-tying term is greater than the ion inertia term (the $\omega^{2}$ in Eq. (70)), and is important when $\mathrm{kv}_{i \perp} / \omega_{c i} \simeq \alpha 1 / 2$ (when the ion FLR and hot electron terms cancel), but is still small compared to the ion FLR and hot electrons terms when they do not cancel. In this case the maximum growth rate is .

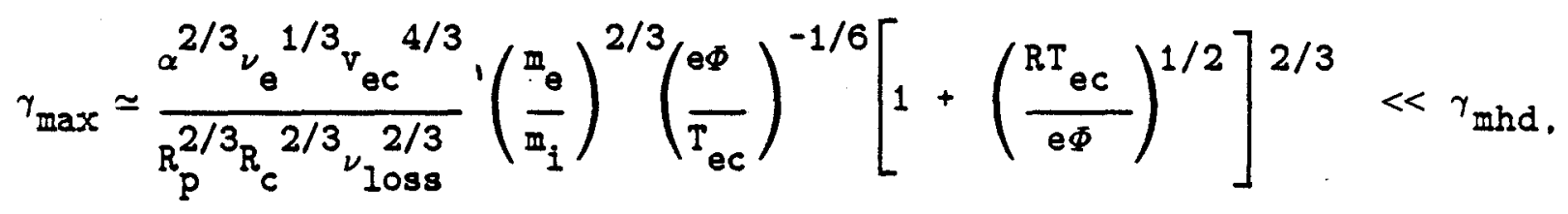

and occurs in a narrow range around $k v_{i \perp} / \omega_{c i}=\alpha^{1 / 2}$. viz.

$$
\left|k v_{i \perp} / \omega_{c i}-\alpha^{1 / 2}\right| \lesssim \frac{v_{i}}{R_{c} \gamma_{\max }}
$$


but well outside this range of $k$ the growth rate is much smaller, given by Eq. (72), except at very low $k$, viz. $k<v_{i \perp}{ }^{2} \bar{\nu}_{l o s s} \omega_{c i} R_{p}^{2} m_{i}^{2} / R_{c} v_{e c}^{4} \nu_{e}^{\alpha^{3} m_{e}^{2}}$, where

$\omega \simeq \frac{\exp (\pi i / 3) \gamma_{\operatorname{mhd}, i}^{4 / 3} \nu_{e}^{1 / 3} k_{\mathrm{vec}}^{4 / 3}}{\bar{\nu}_{\text {loss }}^{2 / 3} \omega_{c i}{ }^{2 / 3} \omega_{c e}{ }^{2 / 3}\left(e \Phi / T_{e}\right)^{1 / 6}}\left[1+\left(\frac{\mathrm{RT}}{e c}\right)^{1 / 2}\right]^{2 / 3}$

(If this $\omega$ is less than $\nu_{e}$, then Eq. (75) must be modified). At still higher ion temperatures,

$$
T_{i}>\frac{T_{e c} \alpha^{7 / 3} \nu_{e}^{2 / 3} v_{e c}^{2 / 3} R_{e}^{2 / 3}}{R_{p}^{4 / 3} \bar{\nu}_{\text {loss }}^{4 / 3}\left(e \Phi / T_{e c}\right)^{1 / 3}}\left(\frac{m_{i}}{m_{e}}\right)^{1 / 3}\left[1+\left(\frac{R T_{e c}}{e \Phi}\right)^{1 / 2}\right]^{4 / 3}
$$

or $\mathrm{T}_{\mathrm{i}}>1 \mathrm{keV}$ for Tara parameters, the line-tying term is important over a broad range of $k$, up to

$k_{\max } \simeq \frac{R_{p}^{2 / 7} \omega_{c i} \vec{\nu}_{10 s s^{2 / 7}}}{R_{c}^{1 / 7} v_{i}^{4 / 7} \nu_{e}^{1 / 7} v_{e c}^{4 / 7}}\left(\frac{m_{i}}{m_{e}}\right)^{2 / 7}\left(\frac{e \Phi}{T_{e c}}\right)^{-1 / 14}\left[1+\left(\frac{R_{e c}}{e \Phi}\right)^{1 / 2}\right]^{2 / 7}$ 
In this regime. Eq. (75) is valid for $k \ll k_{\max }$. Eq. (72) is valid (with $\left.\left|\omega_{* i}\right| \gg \alpha \omega_{c i} / k R_{p}\right)$ for $\omega_{c i} / v_{i} \gg k \gg k_{\max }$ and the maximum growth rate $\gamma_{\max } \simeq \frac{v_{i \perp}^{4 / 7} \nu_{e}^{1 / 7} v_{e c}^{4 / 7}}{R_{p}^{2 / 7} R_{c}^{6 / 7} \bar{\nu}_{l o s s}^{2 / 7}}\left(\frac{m_{e}}{m_{i}}\right)^{2 / 7}\left(\frac{e \Phi}{T_{e c}}\right)^{-1 / 14}\left[1+\left(\frac{R T_{e c}}{e \Phi}\right)^{1 / 2}\right]^{2 / 7} \ll \gamma_{\text {mhd }, i}$

occurs at $k \simeq k_{\max }$. (For $k v_{i \perp} / \omega_{c i}>1$. Eq. (72) shows $\omega<\omega_{d i}$, and Eq. (70) is not valid). The hot electron term is always negligible.

All of the results following from Eq. (70) are only valid if $\omega \gg \omega_{\mathrm{di}}$, and $\mathrm{kv}_{i \perp} / \omega_{c i} \ll 1$. These assumptions are both violated, for the fastest growing modes, if the ion temperature is very high, viz.

$T_{i} \gtrsim \frac{T_{e c} R_{c}^{2 / 3} \nu_{e}^{2 / 3} v_{e c}^{2 / 3}}{R_{p}^{4 / 3} \nu_{\text {loss }}^{4 / 3}}\left(\frac{m_{e}}{m_{i}}\right)^{1 / 3}\left(\frac{e \Phi}{T_{e c}}\right)^{-1 / 3}\left[1+\left(\frac{R_{e c}}{e \Phi}\right)^{1 / 2}\right]^{4 / 3}$

or $T_{i} \gtrsim 20 \mathrm{keV}$ for Tara parameters. In this case we must use Eq. (37) for the dispersion relation, but we may neglect the hot electron term. 
The growth rate and real frequency of the ion driven mode as a function. of $k$ have been found numerically for various values of $T_{i}, \alpha$, and $n_{0}$ (where we set $\bar{\nu}_{\text {Ioss }}=n_{0}\langle\sigma v\rangle$, and set $\left\langle\sigma v=10^{-8} \mathrm{~cm}^{3} \mathrm{sec}^{-1}\right)$. Figure 5 shows the case $T_{i}=100 \mathrm{eV}, \mathrm{n}_{0}=3 \times 10^{10} \mathrm{~cm}^{-3}, \alpha=0.1$. This case falls into the low $\mathrm{T}_{i}$ regime defined by $\mathrm{Eq} .(71)$. The maximum growth rate is $\gamma_{\text {mhd, } i \text {, but }}$ outside of a narrow range of $m\left(\equiv k R_{p}\right)$, from 9 to 15 , the growth rate is very small. Figure 6 shows the case $\mathrm{T}_{i}=100 \mathrm{eV}, \mathrm{n}_{0}=3 \times 10^{11} \mathrm{~cm}^{-3}, \alpha=$ 0.1. where the maximum growth rate should be given by Eq. (73). Here $\gamma_{\max }<$ $\gamma_{\text {mhd, } i}$, and again the modes have growth rates comparable to $\gamma_{\max }$ only over a limited range of $\mathrm{m}$. from 8 to 17 . Figure 7 shows the case $\mathrm{T}_{i}=5 \mathrm{keV}, \mathrm{n}_{0}=$ $3 \times 10^{11} \mathrm{~cm}{ }^{-3}, \alpha=0.1$, where the maximum growth rate should be given by Eq. (78). Here the instability occurs over a broad range of $m$, from 1 to 3 , but the growth rate is much less than $\gamma_{\text {mhd, }}$. In all of these cases we might expect the ion driven modes to saturate nonlinearly at a low level, and not to be harmful to confinement, because they have $\gamma \ll \gamma_{\text {mhd, } i}$, or because the instability is worst at high $m$. This is consistent with the fact that these modes do not seem to affect confinement in Tara, although the $m=1$ mode may have observed at a low amplitude. ${ }^{6}$ However, the modes are linearly unstable. so it is possible that they will adversely affect confinement in some regime. 
Finally. Fig. 8 shows the case $\mathrm{T}_{i}=20 \mathrm{keV}, \mathrm{n}_{0}=3 \times 10^{11} \mathrm{~cm}^{-3}, \alpha=$ 0.1 . In this case, $\omega<\hat{\omega}_{\mathrm{d} I}$ for all modes numbers; the $\mathrm{m}=1$ mode is marginally stable, while all modes with m $>1$ are damped. Thus we expect the ion driven mode to be completely stable when $T_{i}>20 \mathrm{keV}$. for $\mathrm{n}_{0}=3 \times$ $10^{11} \mathrm{~cm}^{-3}$. From the stability analysis of the hot electron driven mode described in Sec. III, we would also expect the hot electron driven mode to be stable for all $\mathrm{m}$, at $T_{i}=20 \mathrm{keV}$ and $\mathrm{n}_{0}=3 \times 10^{11} \mathrm{~cm}^{-3}$, if $\alpha<0.3$ (compare Fig. 3). However, the marginal stability curves given in Figs. 3 and 4 are based on a dispersion relation. Eq. (36), which neglects the ion drive term, and that approximation is starting to break down when $T_{i}=20$ $\mathrm{keV}$ at $\mathrm{n}_{0}=3 \times 10^{11} \mathrm{~cm}^{-3}$. Preliminary calculationis of the hot electron driven mode using Eq. (37), which includes the ion drive, suggest that $\alpha_{\text {crit }}$ for the hot electron driven mode is not much affected by the ion drive term when $T_{i}=20 \mathrm{keV}$ and $n_{0}=3 \times 10^{11} \mathrm{~cm}^{-3}$. but that this mode can be unstable (for sufficiently high $m$ ) even when $\alpha=0$, if $T_{i}$ is somewhat greater, or $n_{0}$ is somewhat smaller. (In this regime, the "hot electron driven mode" is really driven by the ions). Thus, if $\mathrm{n}_{0}=3 \times 10^{11} \mathrm{~cm}^{-3}$ and $\alpha<0.3$, there appears to be a range of $\mathrm{T}_{i}$ (perhaps $20 \mathrm{keV}$ to $50 \mathrm{keV}$ ) where all interchange modes are stable, but this range of stability vanishes if $\mathrm{n}_{0}$ is lower (say $\mathrm{n}_{0}<10^{11} \mathrm{~cm}^{-3}$ ) or $\alpha$ is higher. This conclusion is somewhat uncertain 
because of the preliminary nature of our numerical analysis of the dispersion relation (there may be unstable solutions that we have overlooked), and also because the ion Larmor radius is only about a factor of 3 smaller than the plasma radius at $T_{i}=20 \mathrm{keV}$, so the local approximation, on which the dispersion relation is based, may not be valid. 
V. Summary and Conclusions

A dispersion relation has been found for low frequency interchange modes $\left(\omega \ll \omega_{c i}\right)$ in a plasma with hot electrons (which can have curvature drift frequency greater than the wave frequency $\omega$ ) and including the effects of bulk line-tying to cold (non-emitting) conducting end walls, using a slab model and the local approximation. We have found that hot electron interchange modes are more easily stabilized than MHD interchange modes for two reasons: 1) if $\omega$ is much greater than the electron collision frequency $\nu_{e}$ which is often true with hot electron interchange modes, the line-tying is enhanced by a factor of about $\left(\omega / \nu_{e}\right)^{1 / 2}$ : because only a thin layer of cold electrons near the loss boundary, of width $\Delta v \simeq\left(T_{e h} / m_{e}\right)^{1 / 2}\left(\nu_{e} / \omega\right)^{1 / 2}$ in velocity space, can respond to the wave; 2) if the hot electrons have a spread of drift velocities, then those electrons whose drift velocity $\omega_{d}\left(v_{\perp}, v_{\|}\right)$is resonant with the wave $\left(\omega=\omega_{d}\right)$ can provide a negative dissipation, which, together with the positive dissipative effect of linetying to a cold end wall, can completely stabilize hot electron interchange modes. This result is in contrast to MHD interchange modes, which have $\omega>>$ $\omega_{d}$, and can have their growth rates reduced by line-tying, but cannot be completely stabilized. 
The fraction $\alpha$ of hot electrons needed for marginal stability has been found as a function of azimuthal mode number m (defined as $k R_{p}$ in the slab model). for a range of ion temperatures $T_{i}$ and neutral densities $n_{0}$ relevant to the Tara tandem mirror. (The neutral density enters because the electron end loss rate, which affects the line-tying, is assumed to be equal to the ionization rate $\left.n_{0}<\sigma v\right\rangle$.) We find that the $\alpha$ needed for marginal stability reaches a minimum (called $\alpha_{\text {crit }}$ ) at an intermediate value of m (called m crit , because lower m modes are more effectively stabilized by line-tying. and higher m are more decoupled from the hot electrons (as well as being stabilized by ion FLR, if $\left.T_{i}>1 \mathrm{keV}\right)$. For $T_{i}=100 \mathrm{eV}$ and $n_{0}=3 \times 10^{11}$ $\mathrm{cm}^{-3}$ (corresponding to $10^{-5}$ torr of gas pressure), which are typical of Tara operation without neutral beams, and using other parameters appropriate to Tara, we find $\alpha_{\text {crit }}=0.12$ and $\mathrm{m}_{\text {crit }}=5$. These results are in fairly good agreement with the observed fraction of hot electrons at the onset of instability in the Tara plug, and the observed values of In at which the instability occurs, provided a reasonable estimate is made of the relative mode amplitude in the central cell and plug. (Our model assumes the mode is flute-like, but the conditions for the mode to be flute-like are not satisfied in Tara, and we estimate that the mode amplitude is 5 or 10 times higher in the plug than in the central cell). At $T_{i}=10 \mathrm{keV}$, appropriate for the Tara plug with neutral beams, merit is somewhat lower (because ion FLR stabilizes the higher m modes), and $\alpha_{\text {crit }}$ is somewhat higher, but not 
very much higher: ion FLR has only a weak stabilizing effect. At lower neutral density, $n_{0}=3 \times 10^{10} \mathrm{~cm}^{-3}$. Iine-tying is of course weaker, and both $\alpha_{c r i t}$ and $m_{c r i t}$ are lower (because line-tying preferentially stabilizes low Il modes). This is also consistent with obervations on Tara ${ }^{6}$, which suggest that the hot electron interchange modes have lower m when the neutral density is lower.

At the very least, these results show qualitatively that line-tying should be important in Tara (as well as in TMX-U and EBT, which have somewhat comparable parameters), and indicate the need for more sophisticated models which can be compared quantitatively with experiments. In EBT, the outer field lines can be described as a single cell mirror with end walls at the mirror throats. so the assumption that the modes are flutelike should be valid. However, the ion term should be generalized to $\omega$ $>\omega_{c i}$ (which is simple to do for cold ions), and the line-tying term should be derived for $B_{\text {wall }}=B_{\max }$, instead of $B_{\text {wall }} \ll B_{0}$ as we have assumed. Hot electron interchange modes in EBT are observed ${ }^{7}$ at $m \simeq 7$, and the lower $m$ modes may well be stabilized by line-tying. For Tara, the most important change to be made in the model is to allow the mode to have a different amplitude in each cell (central cell, plug, anchor, fan) so that the modes will be hot electron trapped particle modes rather than interchange modes. Work on this model is in progress. In the central cell of Tara, the neutral density is much greater on the outer field lines than it is on axis, so a 
radial normal mode analysis may be necessary, rather than making use of the local approximation. At the higher ion temperatures expected when neutral beams are used, $\beta$ in the plug should be significant, and electromagnetic effect (coupling between shear and compressional motion of field lines, bending of field lines) could be important; these should be included in the model. When $\omega \gg \nu_{\theta}$. nonlinear effects can be important if the perturbed potential $\phi^{(1)}$ is greater than the width of the boundary layer, which is $\left(\nu_{e} / \omega\right)^{1 / 2} \Phi \simeq 10 \mathrm{~V}$ in Tara. If $\phi^{(1)}$ is much less than $\Phi$, an analytic treatment of the nonlinear behavior may be feasible. The modes observed in Tara have amplitudes in this range, so it would be of interest to see whether such an analytic nonlinear model would predict a saturation amplitude in agreement with the observations.

Our theory has also been compared to the results of Brown et.al. 27 . who measured the hot electron fraction $\alpha$ needed for marginal stability in a single cell mirror experiment. Their results were in good agreement with our theory (within a factor of 2), at low neutral gas densities, where we believe that classical end losses were dominant. At higher densities, where the agreement was not so good (off by a factor of 3 or 4). there was reason to believe that losses were dominated by an anomalous process, not included in our line-tying model. 
In addition to the hot electron driven interchange modes, we have also found ion driven interchange modes which are unstable even if there are no hot electrons. With low ion tempreature and weak line-tying, these modes can have MHD-like growth rates, but (if there are at least a few percent hot electrons) only over a narrow range of high azimuthal mode numbers m; at lower II the growth rates are much lower. With higher ion temperature and stronger line-tying, the growth rate is a broader function of $m$, but the maximum growth rate is well below the MHD growth rate. In either case, there is reason to hope that these modes will saturate at a low level, and not do much harm to confinement. When the line-tying is strong enough (neutral density $n_{0}>10^{11} \mathrm{~cm}^{-3}$ for Tara parameters) and the hot electron fraction is not too large $(\alpha<0.3$ for Tara). there may be a range of ion temperatures (perhaps $20 \mathrm{keV}$ to $50 \mathrm{keV}$ ) where both the ion driven mode and the hot electron driven mode are stable at all mode numbers $\mathrm{m}$. 


\section{Acknowledgements}

The authors wish to express their deep appreciation to Y.T. Lau for computing the marginal stability curves and growth rates, and to A.J. Lichtenberg for help in analyzing the experimental results described in Ref. 27. We are also grateful to M. Mauel, N. Rynn, S. Hiroe, H.L. Berk, R.H. Cohen, J. Kesner, R.S. Post, and W.M. Nevins for useful discussions. This work was supported by U.S. Department of Energy Contract No. DE-ACO278ET-51013. 


\section{REFERENCES}

1. M. N. Rosenbluth and C. L. Longmire, Ann. Phys. 1. 120 (1957); L. S. Hall and B. McNamara, Phys. Fluids 18. 552 (1975).

2. H. L. Berk, M. N. Rosenbluth, H. V. Wong, T. M. Antonsen, D. E. Baldwin, and B. Lane, in Plasma Physics and Controlled Nuclear Fusion Research, 1982, vol. II, p. 175 (IAEA, Vienna, 1983),

3. D. D. Ryutov and G. V. Stupakor, Pis'ma Zh. Eksp. Teor. Fiz. 26, 186 [JETP Lett. 26, 174 (1977)]; Fiz. Plazmy 4. 501 [Sov. J. Plasma Phys. 4. 278 (1978)]; Dokl. Akad. Nauk. SSSR 240, 1086 [Sov. Phys.- Dokl. 23, 412 (1978)] ; R. H. Cohen, Nucl. Fusion 19, 1579 (1979).

4. J. Kesner, Nucl. Fusion 20, 557 (1980); J.'Kesner, R. S. Post, B. D. McVey, and D. K. Smith, Nucl. Fusion 22, 549 (1982).

5. D. E. Baldwin and B. G. Logan. Phys. Rev. Lett. 43, 131 (1979)

6. M. E. Mauel, et.al., to be submitted to Phys. Fluids, 1985.

7. S. Hiroe, J. B. Wilgen, T. W. Baity, L. A. Berry, R, J. Colchin, W. A. Davis, A. M. El Nadi, G. R. Haste, D. L. Hillis, D. A. Spong, T. Uckan, and T. L. Owens, Phys. Fluids 27. 1019 (1984).

8. N. A. Krall, Phys. Fluids 9, 820 (1966).

9. M. Vuillemin, Nucl. Fusion Supplement, Part I, 341 (1962); G. E. Guest and C. 0. Beasley. Phys. Fluids 9,1798 (1966); B. Lehnert, Phys. Fluids 9. 1367 (1966); M. V. Babykin, P. P. Gavrin, E. K. Zavoiskii. L. I. Rudakov and V. A. Skoryupin, Zh. Eksp. Teor. Fiz. 47, 1631 (1964) [Sov. 
Phys. JETP 20. 1096 (1965)]; M. Cotsaftis, Euratom Report EUR-CEA-FC-311 (July 1965).

10. R. F. Post, R. E. Ellis, F. C. Ford, and M. N. Rosenbluth, Phys. Rev. Lett. 4. $166(1960)$; F. R. Scott, T. H. Jensen, and C. B. Wharton, in Plasma Physics and Controlled Nuclear Fusion Research. Culham, 1965 (IAEA, Vienna, 1966) vol. II, p. 463; F. H. Coensgen, W. F. Cummins, W. E. Nexsen, Jr., and A. E. Sherman, Phys. Fluids 9. 187 (1966).

11. W. A. Perkins and R. J. Post, Phys. Fluids 6, 1537 (1963).

12. W. B. Kunkel and J. U. Guillory, in Proceedings of the Seventh Conference on Phenomena in Ionized Gases, Belgrade, 1965, edited by B. Perovic and D. Tocsic (Gradjevinska Knjiga, Belgrade, Yugoslavia, 1966), vol. II, p. 702 .

13. C. W. Hartman, Phys. Fluids 9. 821 (1966); W. B. Ard, R. A. Dandle, and R. J. Stetson, Phys. Fluids 9, 1498 (1966).

14. S. Fornaca, Phys. Fluids 26, 797 (1983).

15. R. Prater, Phys. Fluids 17, 193 (1974).

16. R. H. Cohen, private communication.

17. E. B. Hooper, Jr., D. E. Baldwin, T. K. Fowler, R. J. Kane, and W. C. Turner, Phys. Fluids 27. 2264 (1984).

18. R. A. Dandl, H. O. Eason, P. H. Edmonds, A. C. England, G. E. Guest, C. L. Hedrick, J. T. Hogan, and J. C. Sprott, in Plasma Physics and Controlled Nuclear Fusion Research, Madison, 1971 (IAEA, Vienna, 1971). vol. II, p. 607. 
19. H. L. Berk, Phys. Fluids 19, 1255 (1976).

20. H. L. Berk, J. W. Van Dam. M. N. Rosenbluth and D. A. Spong. Phys. Fluids 26, 201 (1983).

21. Y.-J. Chen, W.M. Nevins, and G.R. Smith, paper 1 C9 presented at Sherwood Controlled Fusion Theory Conference, April 25-28, 1982, Sante Fe, New Mexico; also, in Proceedings of the Second Workshop on Hot Electron Ring Physics, San Diego, CA, Dec. 1-3, 1981.

22. N. A. Krall and A. W. Trivelpiece, Principles of Plasma Bhysics, McGrawHill, New York, 1973; Eq. 8.16.5.

23. B.D. Fried and S.D. Conte. The Rlasma Dispersien Eunction (Academic Press, New York, 1961).

24. V. P. Pastukhov, Nucl. Fusion 14, 3 (1974).

25. R.H. Cohen, M.E. Rensink, T.A. Cutler, and A.A. Mirin, Nucl. Fusion 18. 1229 (1978).

26. Y.-J. Chen, D.E. Baldwin, J.A. Byers, R.H. Cohen, T.B. Kaiser, W.M. Nevins, L.D. Pearlstein, and H. Ramachandran, paper 1B-1 presented at Sherwood Theory Conference, Madison, Wisconsin, April 15-17, 1985.

27. I.G. Brown, A.J. Lichtenberg, M.A. Lieberman, and N. Convers Wyeth, Phys. Fluids 19, 1203 (1976).

28. A.J. Lichtenberg, private communication. 
Appendix A - Derivation of Perturbed Particle Loss Rates

We analyze here the line-tying term in the regime $\omega \gg \nu_{e}$. The collision operator for electrons near the loss boundary is

$$
C(f)=\nu_{e} \frac{1}{x^{2}} \frac{\partial}{\partial x} f+\frac{1}{2 x} \frac{\partial f}{\partial x}+\frac{1}{x^{3}} \frac{\partial}{\partial \hat{\mu}}\left(1-\hat{\mu}^{2}\right) \frac{\partial f}{\partial \hat{\mu}}
$$

where $f(x, \hat{\mu})$ is the cold electron distribution function,

$$
\begin{aligned}
& x \equiv \frac{v}{v_{0}} \\
& \hat{\mu} \equiv \frac{v}{v} \\
& v_{0} \equiv\left(\frac{2 T_{e c}}{m_{e}}\right)^{1 / 2}
\end{aligned}
$$

and $\nu_{e}$ was defined after Eq. (23). We consider an equilibrium magnetic and potential profile in which the wall is located in a fan region where the magnetic field $B_{w a l l}$ is small compared to the mirror cell field $B_{O}$ (see Fig. (1)). We denote the potential in the mirror cell by $\Phi$, the potential at the mirror throat by $\Phi_{t h}$, and set the potential to be zero at the wall. 
The $(x, \hat{\mu})$ coordinate system is related to the $(\epsilon, \mu)$ coordinate system as follows

$$
\begin{aligned}
& \epsilon=\frac{1}{2} m v^{2}+q \phi=\frac{1}{2} m v_{0}^{2} x^{2}+q \phi \\
& \mu=\frac{1}{2} \frac{m v^{2}}{B}=\frac{1}{2} \frac{m_{0}^{2}}{B}\left(1-\hat{\mu}^{2}\right) .
\end{aligned}
$$

We assume that the density is negligible except in the central region and therefore the collision operator acts only in this region. We therefore specify that the $(x, \hat{\mu})$ coordinate system refers to the velocity and pitch angle of particles while in the central region.

$$
\begin{aligned}
& x \equiv\left(\epsilon-\mathrm{q}_{0}\right)^{1 / 2}\left(\frac{2}{\mathrm{mv}_{0}^{2}}\right)^{1 / 2} \\
& \hat{\mu} \equiv\left(1-\frac{2 \mu \mathrm{B}_{0}}{\mathrm{mv}_{0}^{2}}\right)^{1 / 2}
\end{aligned}
$$

The loss cone boundary in $(x, \hat{\mu})$ coordinates is shown in Fig. (9), where for convenience we define 


$$
\begin{aligned}
& x_{0}^{2}=\frac{e \Phi_{t h}}{T_{e c}} \\
& \hat{x}_{0}^{2}=\frac{e \Phi}{T_{e c}} .
\end{aligned}
$$

Particles in region (a) pass through the mirror cell into the fan while particles in region (b) are trapped in the mirror cell. We assume that the density is higher in the mirror cell than in the fan and that all collisions occur in the mirror cell. The loss boundary of the passing particles is the line $x=\hat{x}_{0}$, assuming $B_{\text {wall }} / B_{0} \ll 1$. The loss boundary of the central cell trapped electrons is

$$
\hat{\mu}^{2}=\frac{\mathrm{R}-1}{\mathrm{R}}+\frac{\mathrm{x}_{0}^{2}}{\mathrm{Rx} \mathrm{x}^{2}} \equiv \hat{\mu}_{*}^{2}(\mathrm{x})
$$

for $x>\hat{x}_{0}$.

The equilibrium loss rate of electrons through the loss boundary is

$$
\frac{\partial}{\partial t} \int \frac{d \ell}{B} n=\int \frac{d \ell}{B} \frac{d^{3} v}{v_{0}^{3}} \bar{C}\left(f_{0}\right)
$$

where $f_{0}$ is the equilibrium distribution function. In our model this reduces to 
68

$$
\begin{aligned}
& \frac{\partial n}{\partial t}=4 \pi \int_{0}^{\infty} d x x^{2} \int_{0}^{1} \mathrm{~d} \hat{\mu} \quad C\left(f_{0}\right) \\
& =\left.\frac{4 \pi \nu}{2 \pi} \int_{\hat{\mu}_{*}}^{1} \mathrm{~d} \hat{\mu} \frac{\partial f_{0}}{\partial \mathrm{x}}\right|^{\mathrm{x}=\hat{x}_{0}}+\left.4 \pi \nu \int_{\hat{x}_{0}}^{\infty} \mathrm{dx}\left[D_{\mathrm{x}}+D_{\hat{\mu}}\right] \frac{\partial f_{0}}{\partial \mathrm{x}}\right|^{\mu=\hat{\mu}_{*}}
\end{aligned}
$$

where

$$
\begin{aligned}
& D_{x}=\frac{x_{0}^{2}}{2 R x^{4}} \frac{1}{\hat{\mu}_{*}} \\
& D_{\hat{\mu}}=\frac{x^{2}}{x_{0}^{2}}\left(1-\frac{x_{0}^{2}}{x^{2}}\right) \hat{\mu}_{*}
\end{aligned}
$$

For $\quad R>1, x \simeq \hat{x}_{0}>x_{0}$

$$
D_{x} \simeq \frac{1}{2 R} \frac{x_{0}^{2}}{x_{0}^{14}}
$$

$$
D_{\hat{\mu}} \simeq \frac{\hat{x}_{0}^{2}}{x_{0}^{2}}
$$


and thus $D_{x} \ll D_{\mu}$.

We now consider the perturbation of the particle loss rate due to the fluctuating potential $\phi$. The perturbed distribution function $f^{(1)}$ can be written

$$
f^{(1)}=q \phi \frac{\partial f_{0}}{\partial \epsilon}+h
$$

where $q=-e$ is the electron charge. In the high bounce frequency limit $h$ is constant along a field line, $h=h(\epsilon, \mu)$ and satisfies

$$
\left(\omega-\bar{\omega}_{d}\right) h=-\left(\omega-\hat{\omega}_{*}\right) q \bar{\phi} \frac{\partial f_{0}}{\partial \epsilon}+i c\left(q \phi \frac{\partial f_{0}}{\partial \epsilon}+h\right) .
$$

where $\epsilon=\frac{1}{2} m v^{2}+q \Phi$ and the overbar signifies a bounce average. In the bulk of the distribution function the collision operator can be ignored giving

$$
h=h_{0} \equiv-\frac{\left(\omega-\hat{\omega}_{*}\right)}{\omega-\bar{\omega}_{d}} q \bar{\phi} \frac{\partial f_{0}}{\partial \epsilon}
$$


In the boundary layer we assume that the quantity $h_{0}$ and the adiabatic response $q_{\bar{\phi}} \partial f_{0} / \partial \epsilon$ vary slowly. We therefore evaluate them at the loss boundary which in $(\epsilon, \mu)$ coordinates corresponds to the line $\epsilon=0$ for passing particles. We note that the term

$$
\hat{\omega}_{*} \frac{\partial f_{0}}{\partial \epsilon} \equiv-\frac{k c}{q B_{0}} \frac{\partial f_{0}}{\partial r}
$$

vanishes at $\epsilon=0$ since $f_{0}$ vanishes at $\epsilon=0$ for all $r$. We further make the assumption that the curvature and grad B drifts are small compared to $\omega$ (or to the $E \times B$ drift frequency $\omega_{E}$ ). Finally, we retain only second derivative terms in the collision operator since we assume the perturbed distribution varies rapidly.

$$
c\left((f)^{(1)}\right)=i \frac{1}{2 x^{3}} \frac{\partial^{2} h}{\partial x^{2}}+\frac{1}{x^{3}}\left(1-\hat{\mu}^{2}\right) \frac{\partial^{2} h}{\partial \hat{\mu}^{2}}
$$

where $\bar{\nu}=\tau_{\text {m }} \nu_{e} /\left(\tau_{\text {m }}+\tau_{f}\right)$. (Here $\tau_{\text {m }}$ is the time an electron near the loss boundary spends in the mirror cell and $\tau_{f}$ is the time the electron spends in the fan.) The contribution of the boundary layer to the perturbed density is given by integrating Eq. (A8) over velocity space. The contribution from the boundary layer is 


$$
\begin{aligned}
& \int_{\substack{\text { boundary } \\
\text { layer }}}^{d^{3} v h}=\frac{i}{\omega-\omega_{E}} \int_{\substack{\text { boundary } \\
\text { layer }}}^{d^{3} v \bar{c}\left(f^{(1)}\right)} \\
& \left.\simeq \frac{i}{\omega} \bar{\nu} \frac{4 \pi}{2 x_{0}} \int_{\hat{\mu}_{*}}^{1} \mathrm{~d} \hat{\mu} \frac{\partial \mathrm{h}}{\partial \mathrm{x}}\right|^{\mathrm{x}=\hat{x}_{0}} \\
& +\left.\frac{i}{\omega} \bar{\nu} 4 \pi \int_{\hat{x}_{0}}^{\infty} \mathrm{dx} \frac{1}{\mathrm{R}} \frac{1}{\mathrm{x}}\left(1-\frac{\mathrm{x}_{0}^{2}}{\mathrm{x}^{2}}\right) \frac{\partial \mathrm{h}}{\partial \hat{\mu}}\right|^{\hat{\mu}=\hat{\mu}_{*}}
\end{aligned}
$$

where the integral proportional to $D_{x} \partial \mathrm{h} / \partial \mathrm{x}$ has been neglected. To proceed we must calculate $h$ in the boundary layer. We first note that at the wall where $B_{\text {wall }}=0$ both the equilibrium and the perturbed potential vanish and that the total particle energy equals the particle's parallel kinetic energy. Since $f^{(1)}(\ell, \epsilon, \mu)$ vanishes at the wall for particles with finite parallel velocities $f^{(1)}$ must vanish on the unperturbed loss boundary $\varepsilon=0$ at the wall, $f^{(1)}\left(l=l_{\text {wall }}, \epsilon=0\right)$. Since $\phi^{(1)}$ vanishes at the wall, $f^{(1)}\left(l=l_{\text {wall }}, \epsilon=0\right)$ is equal to $h(\epsilon=0)$ at the wall and therefore $h(\epsilon, \mu)$ vanishes on the boundary $\epsilon=0$. Note that in the central region this implies that $f^{(1)}=q_{\phi}^{(1)} \partial F_{0} / \partial \epsilon$ on the boundary $\epsilon=0$ which is 
equivalent to forcing $f^{(1)}+F_{0}$ to vanish on the "perturbed" boundary $\epsilon=-q \phi^{(1)}$.

In general the boundary condition on $f^{(1)}(\ell, \epsilon, \mu)$ is that it vanish on the unperturbed $10 s 8$ boundary, $\left(\epsilon_{*}, \mu_{*}\right)$ at the axial point $\ell=\ell_{*}$ where the equilibrium parallel velocity of particles vanishes. $\epsilon_{*}-\mu_{*} \mathrm{~B}\left(\ell=\ell_{*}\right)-\mathrm{q} \phi\left(\ell=\ell_{*}\right)=0$. If $\phi(1)$ had been flute-like through the central region and fan, the boundary condition would be that $h$ vanish along the loss boundary of region (a) which corresponds to particles with zero parallel velocity at the wall. The requirement that $f^{(1)}$ vanish on the boundary of region (b) at the mirror throat, however, implies that $\mathrm{h}=-\mathrm{q}_{0}{ }^{(1)} \partial \mathrm{F}_{0} / \partial \epsilon$, where $\phi_{0}^{(1)}$ is the flute value of $\phi^{(1)}$ in the central region and fan. The loss boundary (b) corresponds to particles confined in the central region with zero parallel velocity at the mirror throat; these particles' parallel energy is not affected by an electrostatic perturbation that is flute-like through both the central region and fan region. For the specific problem at hand we assume that $\phi^{(1)}$ drops to zero before the mirror peak and thus the boundary condition for particles in both region (a) and (b) is that $h(\epsilon, \mu)=0$ along the unperturbed loss boundary. 
Away from the boundary the non-adiabatic perturbed response, $h$, goes over to the collisionless solution $h_{0}$. The appropriate solution near the loss boundary of passing particles therefore, is

$$
h=h_{0}\left\{1-\exp \left[-\left(-2 i \hat{x}_{0}^{3}\left(\omega-\omega_{E}\right) / \bar{\nu}\right)^{1 / 2}\left(\hat{x}_{0}-x\right)\right\}\right.
$$

where the branch cut in the square root is taken along the negative real axis .

Thus the contribution of the passing particle loss boundary to the perturbed density is

$$
\left.\frac{i \bar{\nu}}{\left(\omega-\bar{\omega}_{E}\right)} \frac{4 \pi}{2 \bar{x}_{0}} \int_{\hat{\mu}_{*}}^{1} \mathrm{~d} \hat{\mu} \frac{\partial \mathrm{h}}{\partial \mathrm{x}}\right|^{\mathrm{x}=\hat{x}_{0}}=
$$

$\frac{i}{\left(\omega-\bar{\omega}_{E}\right)}\left(\frac{-2 i\left(\omega-\bar{\omega}_{E}\right) \hat{x}_{0}^{3}}{\bar{\nu}}\right)^{1 / 2} \frac{\omega}{\left(\omega-\bar{\omega}_{E}\right)} \frac{q \bar{\phi}}{2 T_{e c} \hat{x}_{0}} \frac{\tau_{m}}{\tau_{m}+\tau_{f}}\left[\left.\frac{4 \pi \nu}{2 \bar{x}_{0}} \int_{\hat{\mu}_{*}}^{1} \mathrm{~d} \hat{\mu} \frac{\partial f_{0}}{\partial \mathrm{x}}\right|^{x=\hat{x}_{0}}\right]$.

We note that the expression in square brackets is the equilibrium loss rate through the $x=\hat{x}_{0}$ loss boundary. We show in Appendix $B$ that the ratio of the loss rate through this boundary to the total loss rate in the limit $\hat{x}_{0} \gg x_{0}$ and $R \gg 1$ is approximately $\left(1+\left(R T_{e c} / e \Phi\right)^{1 / 2}\right)^{-1}$. Thus the 
contribution to the perturbed density due to the perturbed loss through this boundary (assuming $\tau_{m} \gg \tau_{f}$ ) is

$$
\left.\int_{\substack{\text { poundary } \\ \text { layer }}} d^{3} v \mathrm{~h} \simeq \frac{i \omega}{\left(\omega-\bar{\omega}_{E}\right)^{2}}\left[\frac{-2 i\left(\omega-\omega_{E}\right) \hat{x}_{0}^{3}}{\bar{\nu}}\right]^{1 / 2} \frac{q \bar{\phi}}{2 \mathrm{~T}_{e c} \bar{x}_{0}} \frac{\bar{\nu}_{\text {loss }}}{\left[1+\left(\mathrm{RT}_{\mathrm{ec}} / \mathrm{e \phi}\right)^{1 / 2}\right.}\right]
$$

We now consider the perturbed loss through the boundary $\hat{\mu}=\hat{\mu}_{*}$. Here it is convenient to define the boundary layer variable $\xi=\hat{\mu}-\hat{\mu}_{*}$. Retaining only second derivatives the collision operator in these variables is

$C\left(f^{(1)}\right)=\bar{\nu}\left[\frac{1}{2 x^{3}}\left(\frac{x_{0}^{2}}{\hat{\mu}_{*} R x^{3}}\right)^{2}+\frac{1}{x^{3}} \frac{1}{R}\left(1-\frac{x_{0}^{2}}{x^{2}}\right)\right] \frac{\partial_{f}^{2}}{\partial \xi^{2}}$.

For $x>\hat{x}_{0} \gg x_{0}$ the second term dominates. Thus the solution for $h$ along this boundary is

$$
h=h_{0}\left\{1-\exp \left[(-i \omega / \overline{\nu D} \xi(x))^{1 / 2} \xi\right]\right\}
$$

where

$$
h_{0}=-\frac{q \bar{\phi} \omega}{\omega-\bar{\omega}_{E}} \frac{\partial f_{0}}{\partial \epsilon} \mid \mu=\left(\epsilon-q \Phi_{t h}\right) / B_{\max }
$$




$$
D_{\xi}(x)=\frac{1}{x^{3}} \frac{1}{R}\left(1-\frac{x_{0}^{2}}{x^{2}}\right)
$$

The derivative with respect to $\epsilon$ is taken at constant $r$ and constant $\mu \equiv m v_{\perp}^{2} / 2 B$. Changing back to $(x, \hat{\mu})$ coordinates and using the relation,

$$
\frac{\partial f_{0}}{\partial x}-\frac{\mathrm{x}_{0}^{2}}{\mathrm{Rx}^{3}} \frac{1}{\mu_{*}} \frac{\partial f_{0}}{\partial \mu}=0
$$

on the boundary $\hat{\mu}=\hat{\mu}_{*}(x)$. gives

$$
\left(\frac{\partial f_{0}}{\partial \epsilon}\right)_{\alpha, \mu}=\frac{1}{2 T_{e c} \hat{\mu} R x^{2}}\left(\frac{\partial f_{0}}{\partial \hat{\mu}}\right)_{x, \alpha}
$$

The contribution to the perturbed density from the loss boundary $\hat{\mu}=\hat{\mu}_{*}$ is then.

$$
\begin{aligned}
& \int d^{3} v h=i \int d^{3} v \frac{\bar{\nu}}{\omega-\omega_{E}} c\left(f^{(1)}\right) \\
& \mu=\mu_{*} \\
&\left.\simeq \frac{4 \pi i \bar{\nu}}{\omega-\bar{\omega}_{E}} \int_{\hat{x}_{0}}^{\infty} d x \frac{1}{\bar{R}} \frac{1}{x}\left(1-\frac{x_{0}^{2}}{x^{2}}\right) \frac{\partial h}{\partial \mu}\right|^{\hat{\mu}=\hat{\mu}_{*}}
\end{aligned}
$$




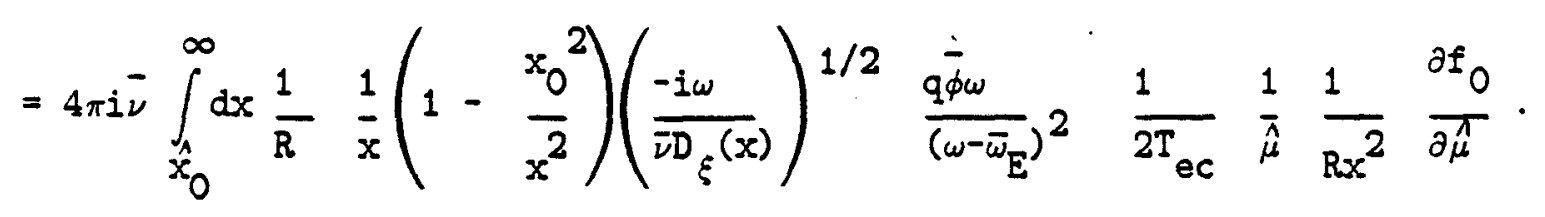

In the limit $\hat{x}_{0}>x_{0}$ this expression is approximately equal to

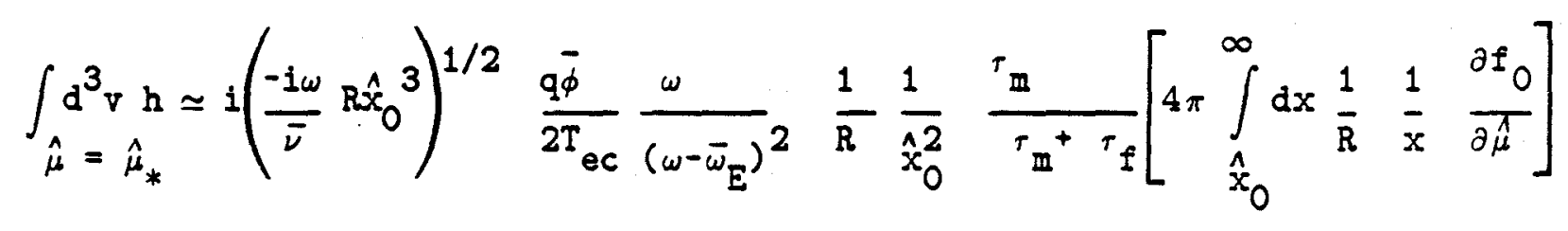

The quantity in brackets is the equilibrium loss through the $\hat{\mu}=\hat{\mu}_{*}$ loss boundary. Thus the order of magnitude of this contribution is

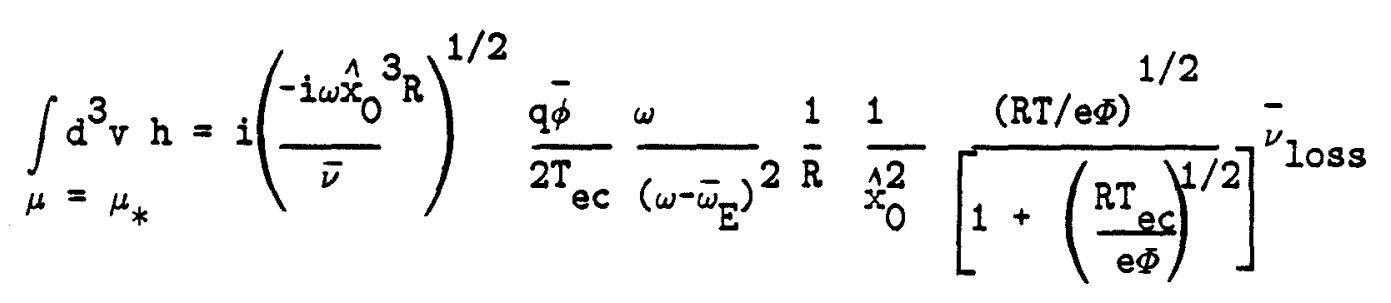

We note that this is smaller than the contribution from the $x=\hat{x}_{0}$ boundary by a factor of $\left(e \Phi / T_{e c}\right)^{-1}$ and we therefore neglect the contribution from the $\hat{\mu}=\hat{\mu}_{*}$ boundary. Thus, the expression for the perturbed particle loss rate is given by Eq. (A15). 
Appendix B - Relative Loss Rates of Mirror Irapped and Passing Species

We justify in this appendix, the estimate made after Eq. (A14) for the relative magnitude of the loss rates for particles loss through boundary (a) and boundary (b). We wish to consider the solution to the equation $C\left(f_{0}\right)=0$ where $C\left(f_{0}\right)$ is given by Eq. (A1). Referring to the phase space shown in Fig. 9 we see that away from the boundary $C\left(f_{0}\right)=0$ is solved by balancing the energy drag with energy diffusion and setting $\partial f_{0} / \partial \mu=0$. Near the boundary the diffusive terms dominate. We can estimate the scale distances, $\Delta x$ and $\Delta \hat{\mu}$, over which this happens by examining when the diffusive terms are comparable to the drag term. Along boundary (a) we estimate

$$
\frac{1}{2 x} \frac{\partial f}{\partial x} \sim \frac{n_{0} \exp \left(-\hat{x}_{0}{ }^{2}\right)}{2 x \Delta x} \sim f \sim n_{0} \exp \left(-\hat{x}_{0}{ }^{2}\right)
$$

which implies

$$
\Delta x \sim \frac{1}{2 x_{0}}
$$

Thus the total loss through boundary of region (a), $\Gamma_{\mathrm{a}}$, is approximately $\nu \mathrm{n}_{0} \exp \left(-\hat{x}_{0}^{2}\right) \mathrm{R}^{-1}$ 
We estimate the slope in $\hat{\mu}$ along the boundary of region (b) by balancing the pitch angle diffusion with energy drag

$$
\frac{1}{x^{2}} \frac{\partial f_{0}}{2 x} \sim \frac{n_{0} \exp \left(-\hat{x}_{0}{ }^{2}\right)}{x_{0}} \sim \frac{1}{\hat{x}_{0}{ }^{3}} \frac{1}{R} \frac{\partial^{2} f_{0}}{\partial \mu^{2}} \sim \frac{1}{\hat{x}_{0}{ }^{3}} \frac{n_{0} \exp \left(-\hat{x}_{0}{ }^{2}\right)}{(\Delta \mu)^{2}}
$$

which gives

$$
\Delta \hat{\mu} \sim \frac{1}{\mathrm{R}^{1 / 2 \hat{x_{0}}}}
$$

Neglecting the energy diffusion through boundary (b) we estimate the length in $x, \Delta x_{b}$, along the boundary (b) over which pitch angle diffusive flow is appreciable. Near the loss boundary the energy and pitch angle diffusion terms balance. This gives

$$
\frac{1}{2 x^{3}} \frac{\partial^{2} f_{0}}{\partial x^{2}} \sim \frac{n_{0} \exp \left(-\hat{x}_{0}{ }^{2}\right)}{2 x^{3}\left(\Delta x_{b}\right)^{2}} \sim \frac{1}{x_{0}^{3}} \frac{1}{R} \frac{n_{0} \exp \left(-\hat{x}_{0}{ }^{2}\right)}{(\Delta \hat{\mu})^{2}}
$$

or

$$
\Delta x_{b} \sim R^{1 / 2} \Delta \mu \sim \frac{1}{x_{0}}
$$


which matches smoothly to the energy scale length along boundary (1) given by Eq. (B-2). Thus the flow through boundary (b) is approximately

$$
\Gamma_{b} \equiv \nu \int_{\hat{x}_{0}}^{\infty} \mathrm{dx} \frac{1}{\bar{x}} \frac{1}{\bar{R}} \frac{\partial f_{0}}{\partial \mu} \sim \nu \Delta x_{b} \frac{1}{x_{0}} \frac{1}{R} \frac{f_{0}}{\Delta \mu} \sim \nu \frac{n_{0} \exp \left(-\hat{x}_{0}{ }^{2}\right)}{\hat{x}_{0} R^{1 / 2}}
$$

Thus the ratio of flow through boundary (a) to the total flow is

$$
\frac{\Gamma_{\mathrm{a}}}{\Gamma_{\mathrm{a}}+\Gamma_{\mathrm{b}}} \sim \frac{1}{1+\frac{\mathrm{R}^{1 / 2}}{\mathrm{x}_{0}}}=\frac{1}{1+\left(\frac{\mathrm{BT}}{\mathrm{e \phi}}\right)^{1 / 2}} .
$$




\section{Figure Captions}

Fig. 1. Axial profiles of the model equilibrium magnetic field $B(z)$, solid line, and the potential $\phi(z)$, dashed line.

Fig. 2 The equilibrium electron distribution function $f_{0}(v)$ and the perturbed electron distribution function $f^{(1)} \underset{\sim}{(v)}$ for $v_{1}=0$. showing the boundary layer of width $\Delta \mathrm{v}$ (defined by Eq. (28)) which occurs when $\omega \gg \nu_{e}$.

Fig. 3. Marginal stability curve of the hot electron driven interchange mode, for high neutral density $\left(n_{0}=3 \times 10^{11} \mathrm{~cm}^{-3}\right)$, for $T_{i}=100 \mathrm{eV}, 1 \mathrm{keV}$ and $10 \mathrm{keV}$.

Fig. 4. Marginal stability curve of the hot electron driven interchange mode, for low neutral density $\left(n_{0}=3 \times 10^{10} \mathrm{~cm}^{-3}\right)$, for $T_{i}=100 \mathrm{eV}, 1 \mathrm{keV}$ and $10 \mathrm{keV}$.

Fig. 5. Real frequency $\omega_{r}$ and growth rate $\gamma$ of ion driven mode, for $n_{0}=3$ $\times 10^{10} \mathrm{~cm}^{-3}, \mathrm{~T}_{i}=100 \mathrm{eV}, \alpha=0.1$.

Fig. 6. Real frequency $\omega_{r}$ and growth rate $\gamma$ of ion driven mode, for $n_{0}=3$ $\times 10^{11} \mathrm{~cm}^{-3}, \mathrm{~T}_{i}=100 \mathrm{eV}, \alpha=0.1$. 
Fig. 7. Real frequency $\omega_{r}$ and growth rate $\gamma$ of ion driven mode, for $\mathrm{n}_{0}=3$ $\times 10^{11} \mathrm{~cm}^{-3}, \mathrm{~T}_{i}=5 \mathrm{keV}, \alpha=0.1$.

Fig. 8. Real frequency $\omega_{r}$ and growth rate $\gamma$ of ion driven mode, for $\mathrm{n}_{0}=3$ $\times 10^{11} \mathrm{~cm}^{-3}, \mathrm{~T}_{i}=20 \mathrm{keV}, \alpha=0.1$.

Fig. 9. Loss boundary (shaded line), electrostatically trapped region (a) and magnetically trapped region $(b)$ in $(x, \hat{\mu})$ phase space. 


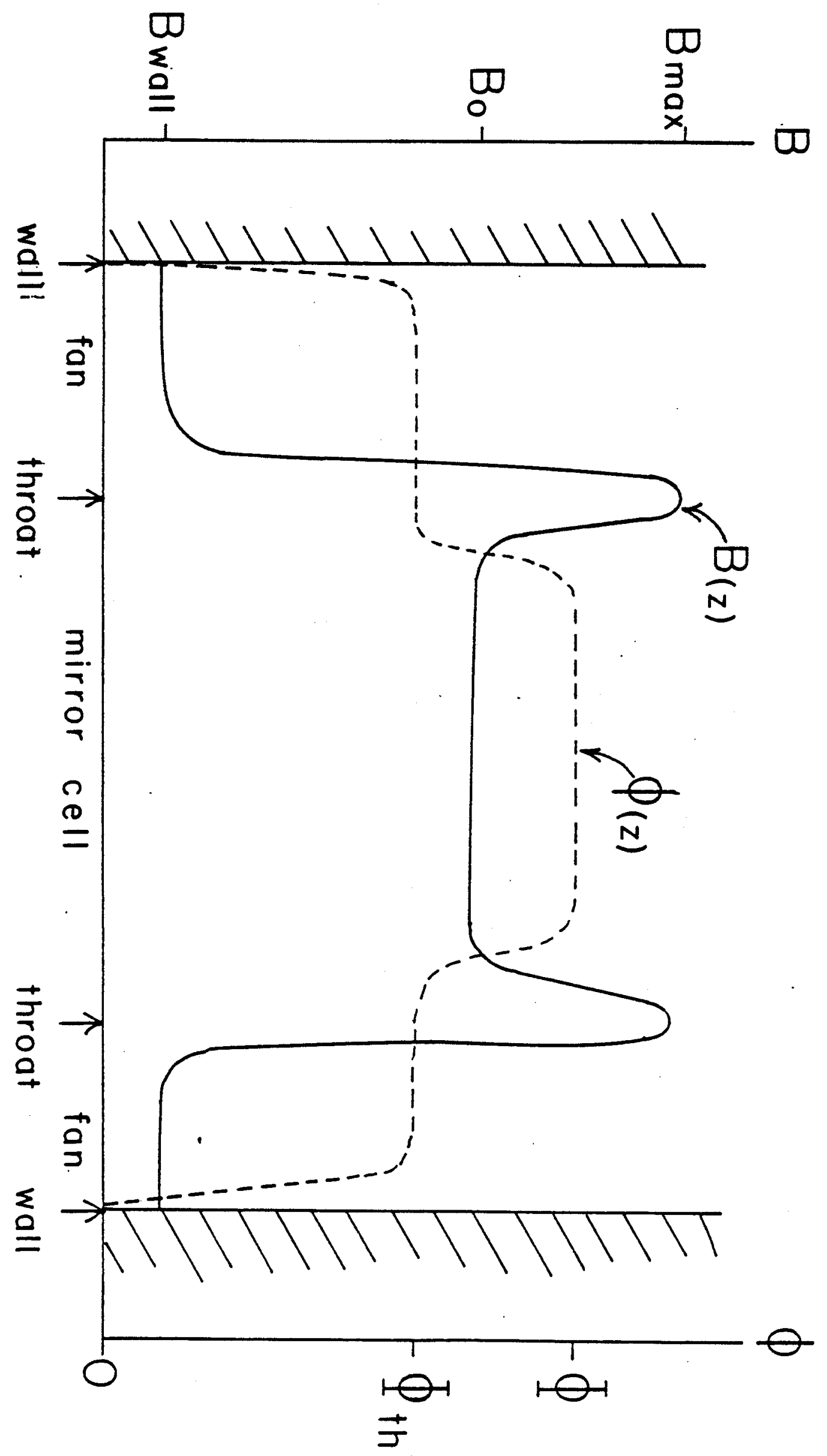




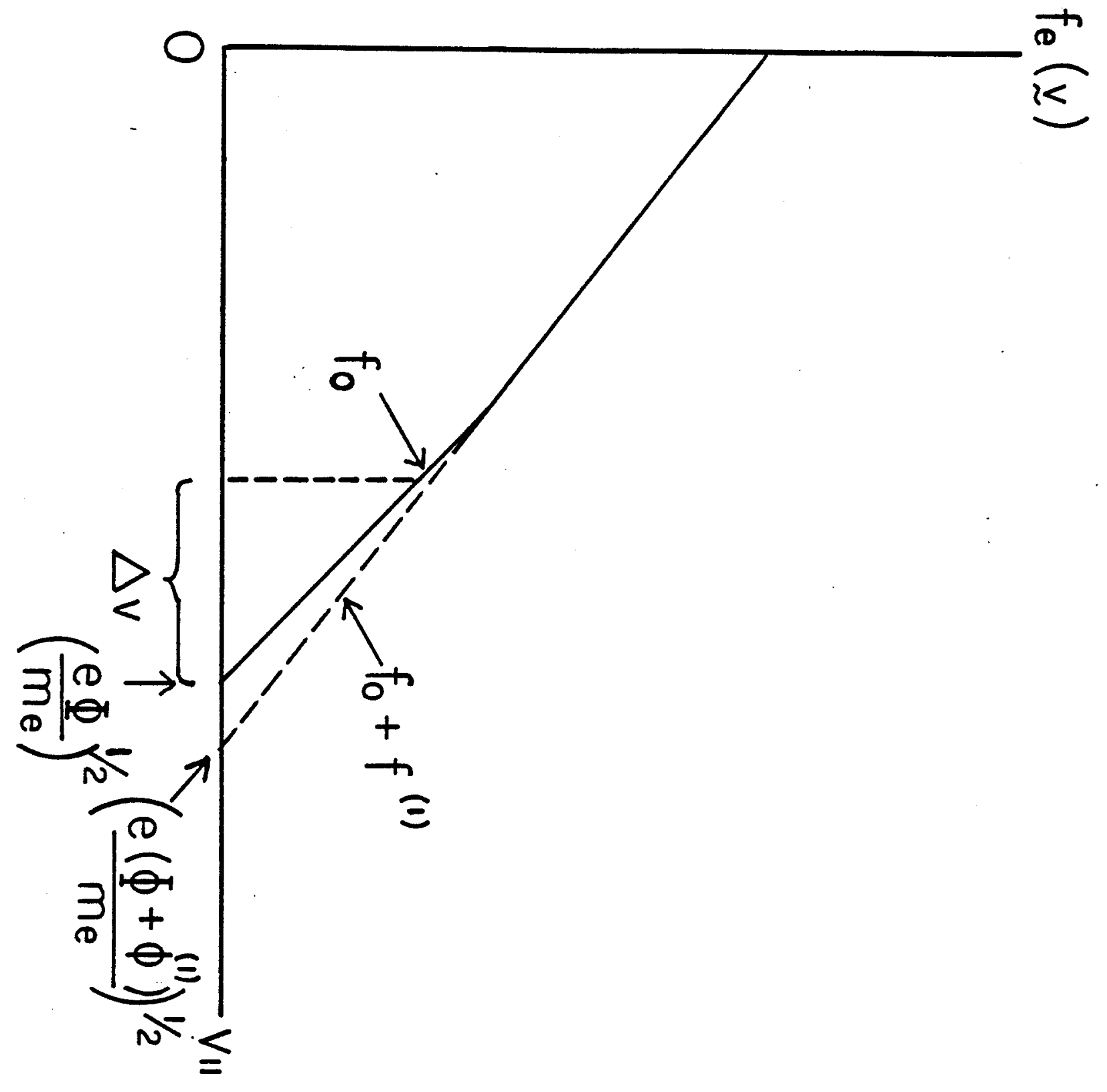


THE MARGINAL INSTABILITY CONDITION OF THE HOT ELECTRON INTERCHANGE MODE

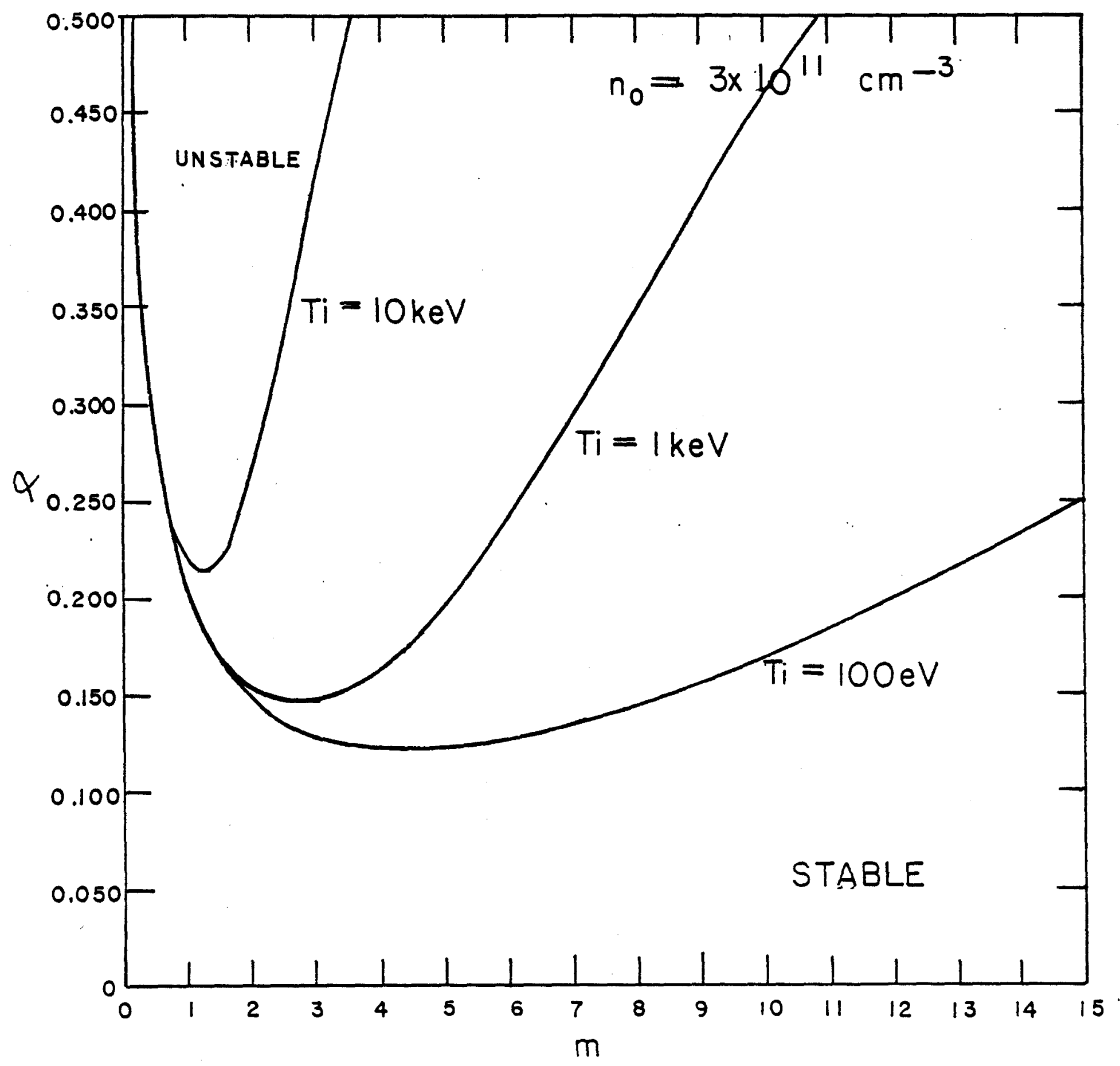


THE MARGINAL INSTABILITY CONDITION OF THE HOT ELECTRON INTERCHANGE MODE

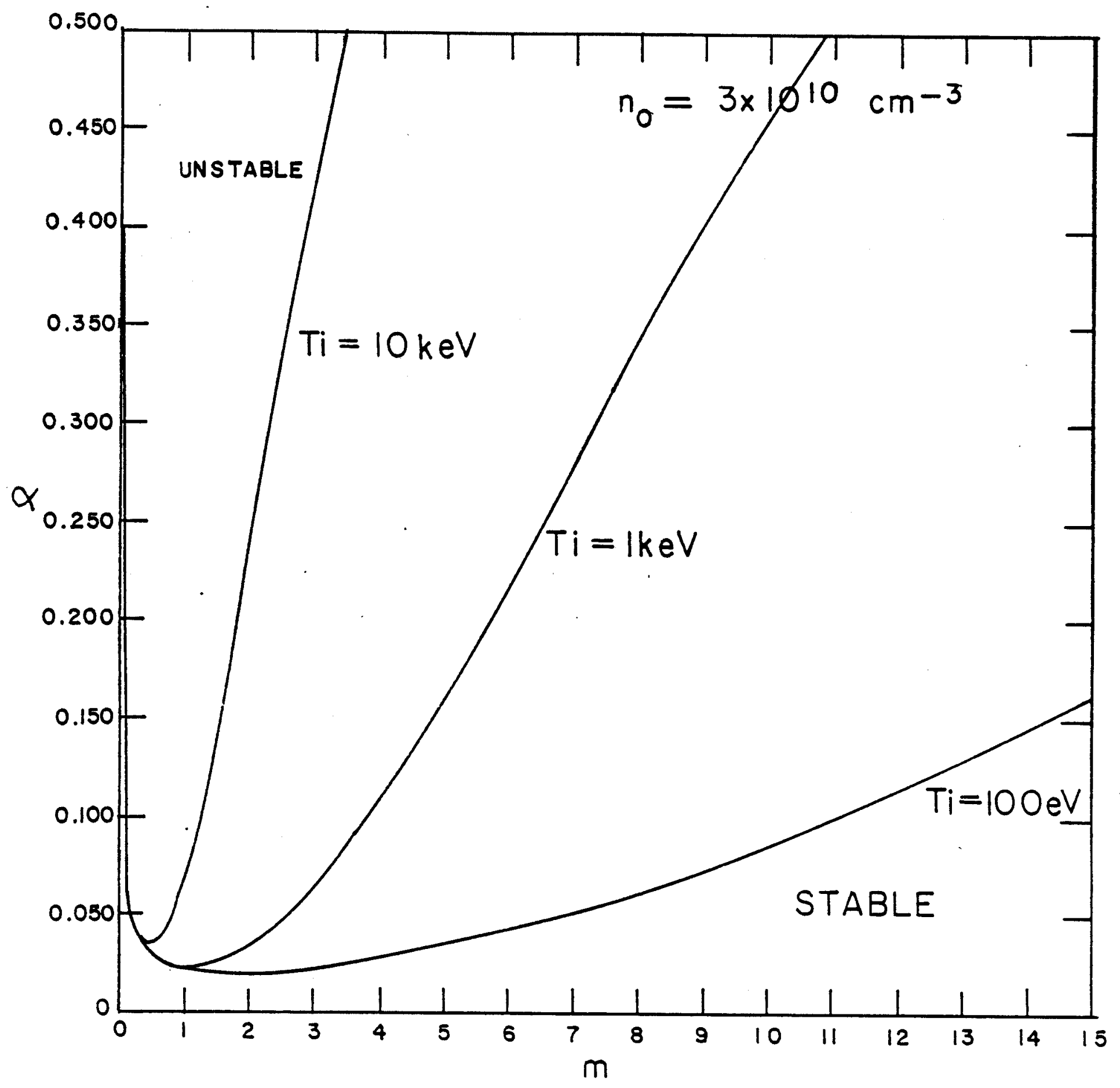


THE DISPERSION RELATION OF
THE ION-DRIVEN MODE

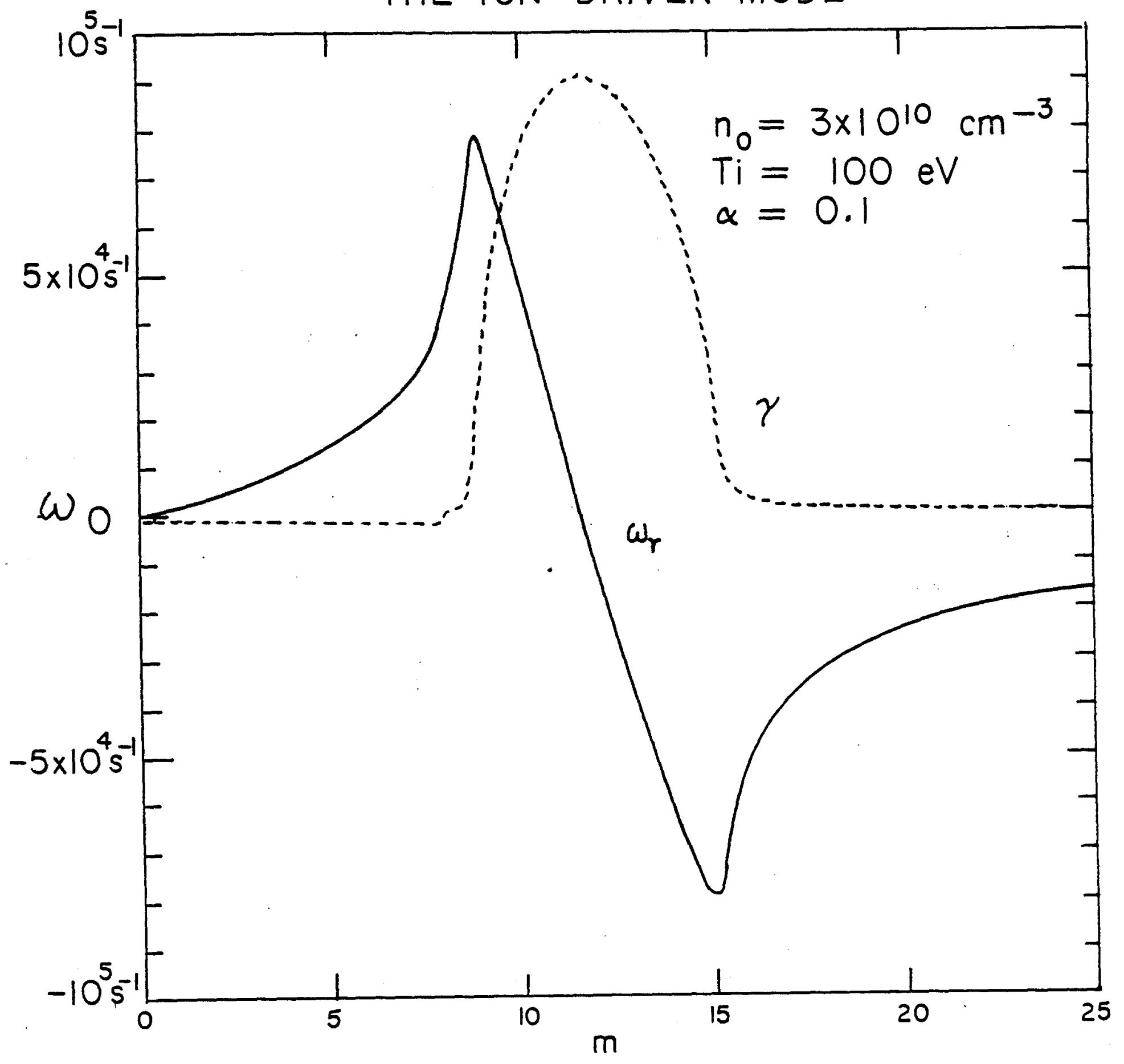




\section{THE DISPERSION RELATION OF THE ION-DRIVEN MODE}

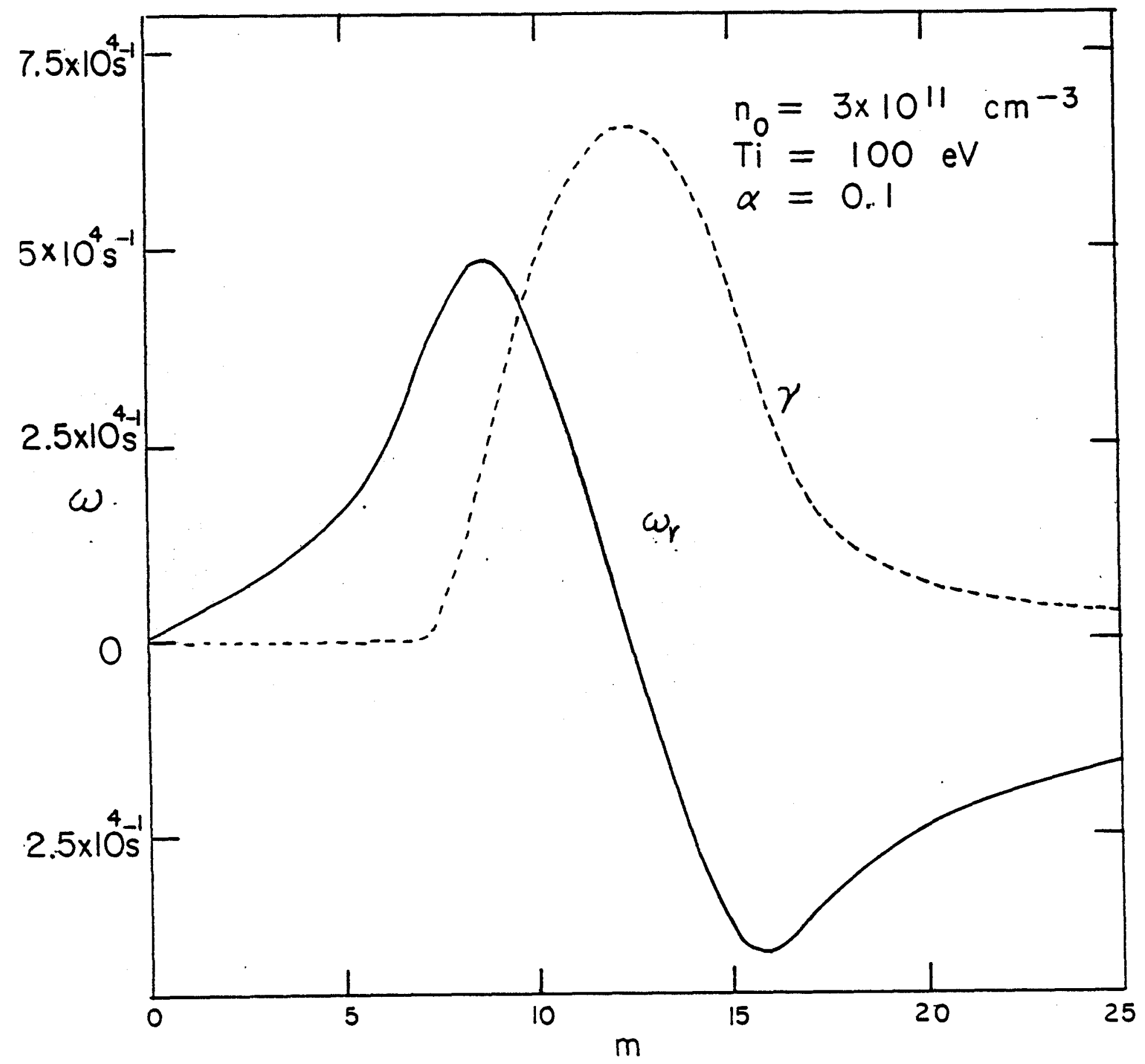




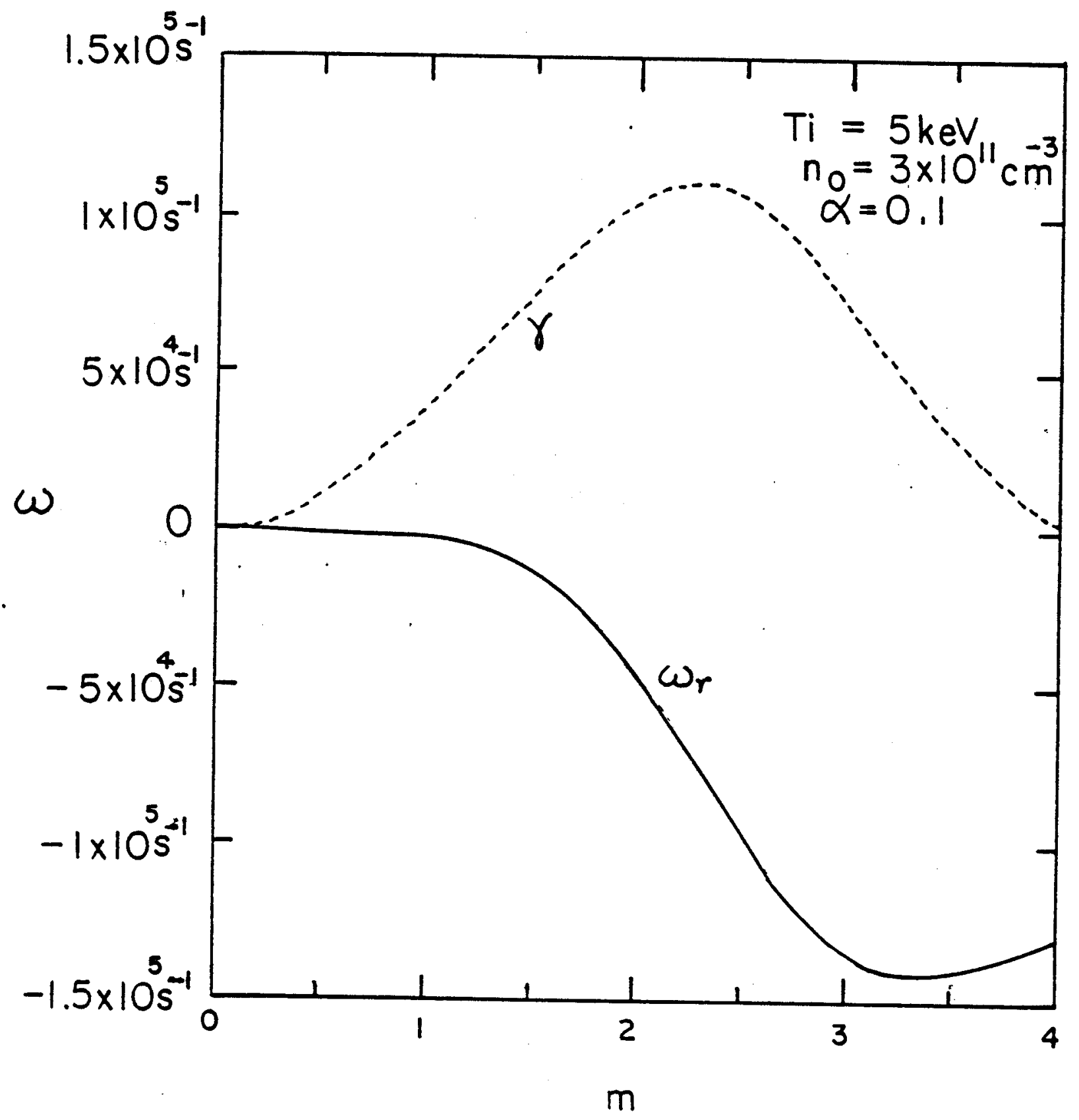




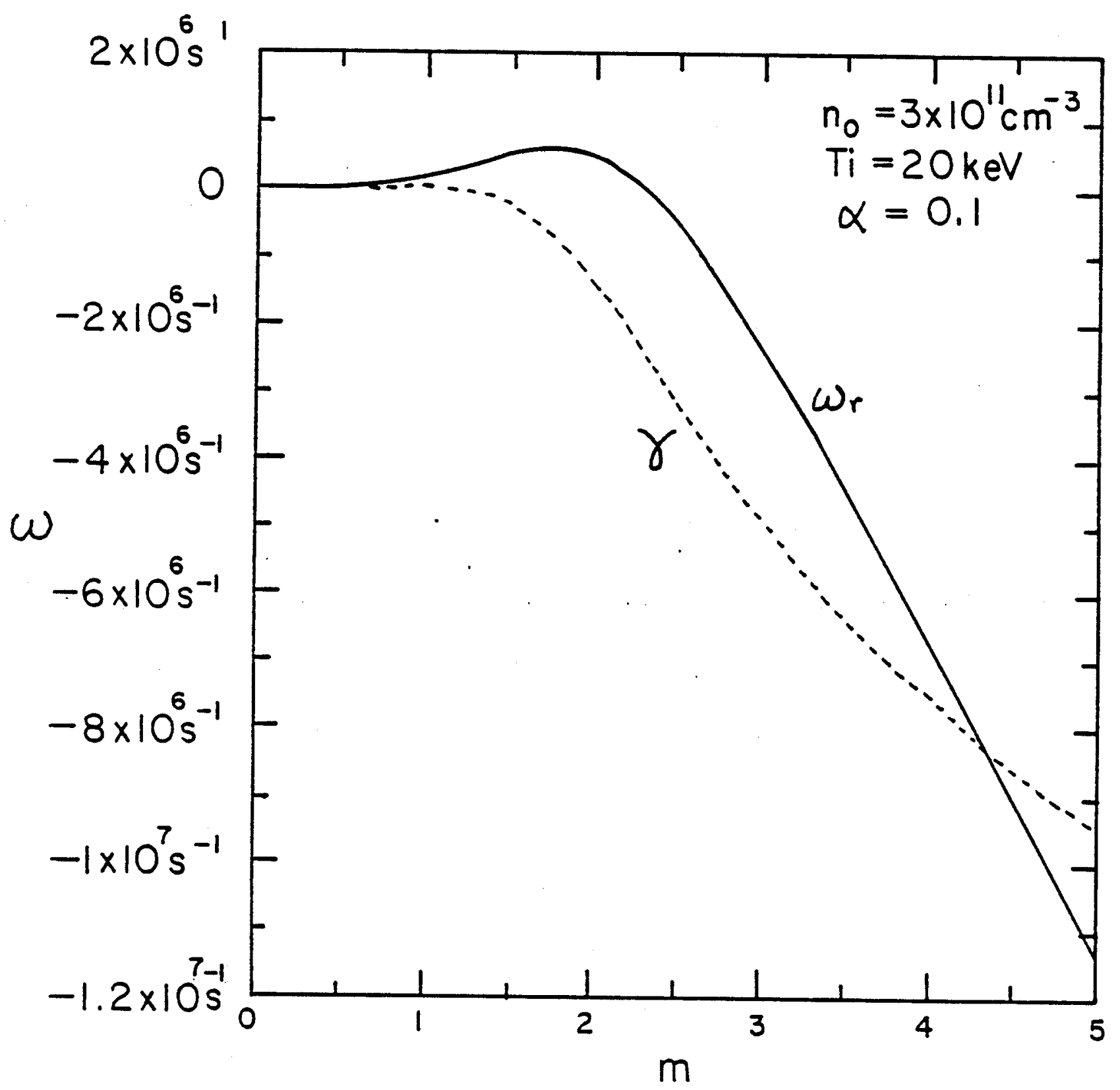




$$
\text { 屑 }
$$

